\title{
2. Die Branchen Textil und Bekleidung
}

\subsection{Ein besonderer Stoff: Textil als Kulturgut und Kommunikationsmittel}

Weil er kein schützendes und wärmendes Fell besitzt, entwickelte der Mensch bereits früh ein starkes Bedürfnis nach Kleidung. Laut der Maslow'schen Theorie zählt dieses zu den Grundbedürfnissen, die wie die Luft zum Atmen oder Essen und Trinken der Lebenserhaltung dienen. So entstand eine der ältesten Kulturtechniken der Menschheit: die Herstellung von Bekleidung und entsprechenden Textilien. Anfangs verwendeten die Menschen zum Schutz vor Kälte und Nässe ausschließlich tierische Felle. Da der Tragekomfort jedoch zu wünschen übrig ließ, experimentierten sie mit tierischen und pflanzlichen Fasern wie Wolle oder Flachs. Später verbesserte die Technik des Nähens die Funktionalität von Kleidung und die spätere Erfindung von Spinn- und Webtechniken ermöglichte es, textile Flächen für die weitere Bearbeitung herzustellen.

Im Laufe der Jahrhunderte wurde Bekleidung aber nicht nur materiell weiterentwickelt, sondern auch zum Kommunikationsmittel, dessen Aussagekraft für Gesellschaften unerlässlich ist. Einst ein Symbol für Reichtum, werden heute über die Kleidung Mitglieder bestimmter Gruppen identifiziert, Unternehmen oder Berufsstände gekennzeichnet. Kleidung gibt Auskunft über Rang und Namen der Tragenden, prägt Stil- und Schönheitsbewusstsein oder ist schlicht ein Statement, indem sie für bestimmte Meinungen, Haltungen oder Stimmungen steht.

Mit der Industrialisierung änderte sich Ende des 18. Jahrhunderts die Textilproduktion in Europa grundlegend. Die englische Erfindung der »Spinning Jenny« ersetzte das Spinnrad und ihre Weiterentwicklung verhundertfachte die Spinnleistung. Der ersten Spinnmaschine folgten rasch automatisierte, dampfmaschinenbetriebene Verfahren. Die deutschen Fabrikanten setzten noch lange auf die billige Handarbeit und gaben den aufkommenden Preisdruck an die Heimarbeiter:innen weiter. Die Folge waren Hungerlöhne trotz endloser Arbeitszeiten und Mitarbeit von Frauen und Kindern. 1840 schrieben schlesische Zeitungen von 50.000 vom Hungertod bedrohten Familien in der Branche. 1844 schlossen sich die Textilbeschäftigten im schlesischen Peterswaldau zum Aufstand gegen die 
Textilfabrikanten Zwanziger und Dierig zusammen. 1892 veröffentlichte Gerhart Hauptmann sein Drama »Die Weber«, das den schlesischen Weberaufstand und die Not der verzweifelten Menschen zum Inhalt hat.

Mitte des 20. Jahrhunderts mussten sich die Textil- und die Bekleidungsbranche einer weiteren grundlegenden Veränderung stellen. Nach zwei Weltkriegen führten steigende Einkommen zu mehr Konsum - auch von textilen Produkten: Auf die »Fresswelle« folgte in Deutschland die »Bekleidungswelle«. Die Kleiderschränke füllten sich, die Bekleidungsproduktion stieg kontinuierlich. Ab Ende der 1960er Jahre beförderten gesellschaftspolitische Veränderungen einen grundsätzlichen textilen Kulturwandel: Klassische Sonntagskleidung wurde im wahrsten Sinne des Wortes »old fashioned «.

Der Anteil des Haushaltseinkommens, der für textile Produkte aufgewendet wurde, ging im Laufe dieser Entwicklung kontinuierlich zurück. Dies hing sowohl mit zunehmenden Bekleidungsimporten aus Niedriglohnländern als auch mit den stetig steigenden Haushaltseinkommen zusammen. Während Anfang der 1960er Jahre über die Hälfte des Einkommens für das leibliche Wohl und das sogenannte »Dach über dem Kopf « ausgegeben wurde (40,3 bzw. 14,3 Prozent; siehe Abbildung 1), wurden für Kleidung und Schuhe immerhin noch 12,1 Prozent des Einkommens aufgewendet. Bis 1998 halbierte sich dieser Haushaltsposten auf 5,7 Prozent, während sich der Anteil für die Wohnung im gleichen Zeitraum mehr als verdoppelte (von 14,3 auf 32,2 Prozent). 
Abbildung 1: Aufwendungen privater Haushalte (1962/1963-1998)

\begin{tabular}{|l|c|c|c|c|c|c|c|c|}
\hline & $\begin{array}{c}1962 / \\
1963\end{array}$ & 1969 & 1973 & 1978 & 1983 & 1988 & $1993^{*}$ & $1998 *$ \\
\hline $\begin{array}{l}\text { hochgerechnete } \\
\text { Haushalte } \\
\text { (in 1.000) }\end{array}$ & 19.659 & 20.540 & 21.155 & 22.050 & 23.469 & 24.877 & 28.917 & 25.954 \\
\hline $\begin{array}{l}\text { Nahrungsmittel, } \\
\text { Getränke, } \\
\text { Tabakwaren }\end{array}$ & $40,3 \%$ & $33,2 \%$ & $28,1 \%$ & $25,5 \%$ & $23,6 \%$ & $22,5 \%$ & $21,0 \%$ & $13,7 \%$ \\
\hline $\begin{array}{l}\text { Bekleidung, } \\
\text { Schuhe }\end{array}$ & $12,1 \%$ & $10,8 \%$ & $10,3 \%$ & $9,2 \%$ & $8,8 \%$ & $8,2 \%$ & $7,1 \%$ & $5,7 \%$ \\
\hline $\begin{array}{l}\text { Miete, Energie, } \\
\text { Wohninstand- } \\
\text { haltung }\end{array}$ & $14,3 \%$ & $18,3 \%$ & $20,5 \%$ & $21,5 \%$ & $24,8 \%$ & $27,4 \%$ & $26,4 \%$ & $32,2 \%$ \\
\hline $\begin{array}{l}\text { Haushalt, } \\
\text { Innenausstattung }\end{array}$ & $11,5 \%$ & $11,9 \%$ & $12,1 \%$ & $11,2 \%$ & $9,4 \%$ & $8,7 \%$ & $8,4 \%$ & $6,8 \%$ \\
\hline \begin{tabular}{l} 
Verkehr \\
\hline Gesundheit
\end{tabular} & $7,7 \%$ & $11,3 \%$ & $13,1 \%$ & $16,0 \%$ & $16,2 \%$ & $15,1 \%$ & $17,1 \%$ & $13,5 \%$ \\
\hline $\begin{array}{l}\text { Bildung, Unter- } \\
\text { haltung, Kultur }\end{array}$ & $6,5 \%$ & $6,5 \%$ & $7,6 \%$ & $7,9 \%$ & $8,4 \%$ & $8,8 \%$ & $8,8 \%$ & $19,9 \%$ \\
\hline $\begin{array}{l}\text { persönliche } \\
\text { Ausstattung }\end{array}$ & $2,8 \%$ & $3,8 \%$ & $3,8 \%$ & $4,5 \%$ & $4,4 \%$ & $4,7 \%$ & $4,3 \%$ & $4,3 \%$ \\
\hline
\end{tabular}

* bis 1988 früheres Bundesgebiet, ab 1993 westdeutsche Bundesländer

Eigene Darstellung; Datenquelle: Statistisches Bundesamt (Destatis): Einkommens- und Verbrauchsstichprobe (EVS; verschiedene Jahrgänge).

Da auch die Aufwendungen für Nahrungs- und Genussmittel im Laufe der Jahre deutlich zurückgingen, konnten die Verbraucher:innen das verbleibende Geld für Konsumgüter und Dienstleistungen ausgeben. Die heimische Textil- und Bekleidungsindustrie schaffte es nicht, die steigende Kaufkraft der privaten Haushalte für sich zu nutzen.

\subsubsection{Branchendefinitionen}

Die Textilindustrie des 20. Jahrhunderts kann grob in drei Produktionsstufen unterteilt werden (siehe Abbildung 2): 
- Erstens die Erzeugung von Garnen aus tierischen, pflanzlichen, mineralischen oder chemischen Rohstoffen durch Spinnen, Zwirnen, Seilen und Texturieren.

- Als nächste Stufe folgt die Herstellung von Flächengebilden, insbesondere zu Geweben, Maschenwaren oder Vliesstoffen. Garne können auch zu Körpergebilden wie Schläuchen oder Strümpfen oder zu Vorerzeugnissen beispielsweise für den Kunststoffbau weiterverarbeitet werden.

- Integriert oder extern organisiert ist die abschließende dritte Stufe, die Textilveredelung durch Bleichen, Färben, Bedrucken oder Ausrüsten. Letzteres ermöglich spezielle Stoffeigenschaften (Griffigkeit, Bügelfreiheit, Verhindern des Einlaufens) oder verstärkt diese.

\section{Abbildung 2: Produktionsstufen der Textil- und der Bekleidungsindustrie}

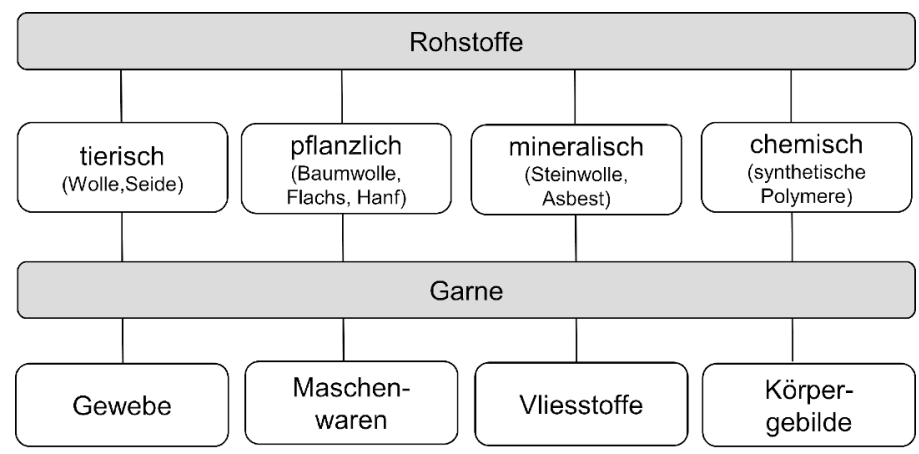

\section{Veredelung}

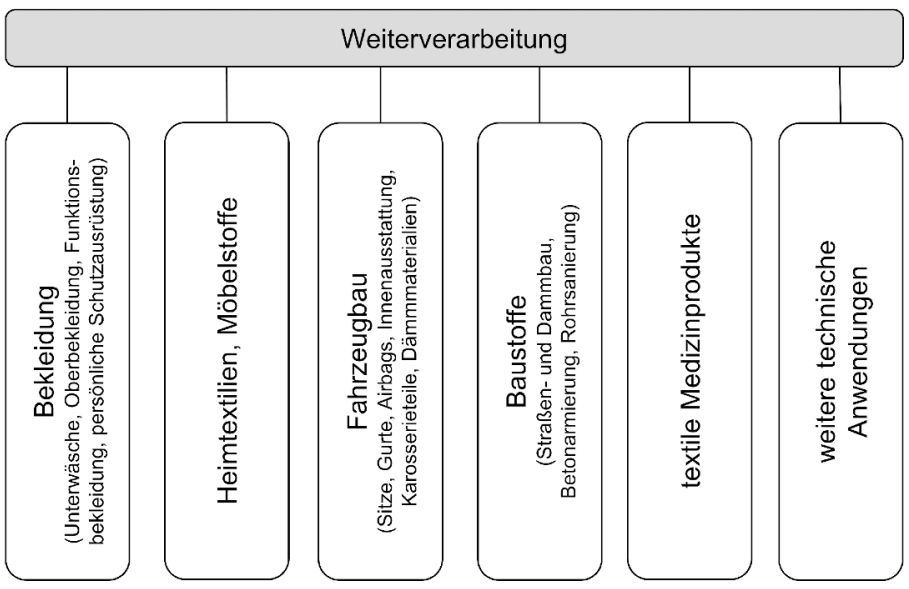

Quelle: Eigene Darstellung. 
Die sogenannten technischen Textilien entwickelten sich im 20. Jahrhundert zu den innovativsten textilen Produkten. Von 1980 bis 1990 stieg der Anteil technischer Textilien an der westeuropäischen Textilproduktion von 9 Prozent auf 22 Prozent; ${ }^{1}$ ihr Umsatzanteil ist inzwischen nach Angaben des "Industrieverbandes Veredelung - Garne - Gewebe - Technische Textilien« (IVGT) auf 50 Prozent aller in Deutschland hergestellten Textilien gestiegen. ${ }^{2}$ Ihre Anwendungsgebiete sind vielfältig; die landläufig bekannte Verarbeitung zu Arbeitsschutzkleidung oder modischer und funktioneller Outdoor-Bekleidung stellt nur einen kleinen Teil davon dar. Technische Textilien kommen auch in der Baubranche als textilbewehrter Beton oder in der Medizin als textile Implantate zum Einsatz. In Flugzeugflügeln und im Fahrzeugbau werden Faserverbundstoffe verarbeitet; auch selbstklebende Teppiche sind hochwertige textile High-Tech-Produkte.

Die Bekleidungsindustrie entstand aus der Entwicklung von handwerklicher Einzelfertigung hin zu einer an industrieller Massenproduktionen orientierten Herstellung. Standardisierte Konfektionsgrößen waren das Fundament, um in größeren Stückzahlen produzieren zu können. Zum Zuschneiden werden viele Stofflagen übereinandergelegt und auch das Nähen und Bügeln sowie die Qualitätskontrolle erfolgt in großen Mengen. Die Näharbeiten werden hochgradig arbeitsteilig vorgenommen, wobei die einzelnen Arbeitsschritte weniger als 30 Sekunden bis zu wenigen Minuten dauern.

Aktuell fertigt die deutsche Bekleidungsindustrie weniger als 5 Prozent der von ihr vertriebenen Produkte im Inland und konzentriert sich in der Regel auf Entwicklung, Einkauf und Vertrieb sowie Logistik und Verwaltung - aber selbst diese organisatorischen Bereiche werden immer stärker ausgelagert.

\subsubsection{Die Struktur der Branchen}

In der Nachkriegszeit arbeiteten noch 17 Prozent aller Industriebeschäftigten in Westdeutschland in der Textil- und der Bekleidungsindustrie. Erst 1973 sank ihr Anteil im Verarbeitenden Gewerbe auf unter 10 Prozent, während der Anteil der Betriebe über diese Zeitspanne hinweg fast stabil bei rund 15 Prozent lag. Im Laufe der nachfolgenden Jahre forderte der Strukturwandel seinen Tribut: 1990 waren nur noch 5 Prozent aller Industriebeschäftigten in der Textil- und der Bekleidungsbranche angestellt und auch die Wiedervereinigung hatte nur wenig Auswirkungen auf den gesamtdeutschen Beschäftigtenanteil: Ende der 1990er Jahre betrug der Wert nur noch 3 Prozent. ${ }^{3}$

\footnotetext{
1 Wikipedia-Artikel »Technische Textilie«, Abschnitt »Historische Entwicklung« (Abruf am 15.1.2021).

2 IVGT (o. J.): Das Netzwerk für die Textilindustrie. Technische Textilien.

3 Statistisches Bundesamt (Destatis) (2008): Fachserie 4, Reihe 4.1.1; eigene Berechnungen.
} 
Die traditionell mittelständisch geprägte betriebliche Struktur änderte sich im Laufe der Zeit kaum: 1951 arbeiteten durchschnittlich 110 Beschäftigte in den Betrieben der Textil- und der Bekleidungsindustrie. Die Rationalisierungsmaßnahmen der nächsten Jahrzehnte reduzierten den Durchschnittswert auf "nur« rund 100 Beschäftigte pro Betrieb und auch nach der deutschen Wiedervereinigung hatten textile Unternehmen im Durchschnitt noch 95 Beschäftigte.

Geografisch blieb die deutsche Textilindustrie seit dem 19. Jahrhundert auf bestimmte Regionen mit unterschiedlichen Produktschwerpunkten beschränkt. Nach dem Zweiten Weltkrieg und dem Verlust der Ostgebiete sowie nach Gründung von Bundesrepublik und DDR siedelten große Unternehmen aus Schlesien, dem Sudetenland und Sachsen nach Westdeutschland um. Damit war ab Beginn der 1950er Jahre die ganze Breite der Textilproduktion in der jungen Bundesrepublik vertreten.

$\mathrm{Zu}$ den traditionellen Textilregionen gehört das Münsterland mit dem Schwerpunkt Baumwollverarbeitung. Konzerne wie NINO, Rawe und van Delden beschäftigten viele Tausend Menschen. Zentrum der Flachsspinnereien und Leinenproduktion war Ostwestfalen, wo sich früh Bekleidungsunternehmen wie Seidensticker, Brax-Leineweber, Hucke und Ahlers mit großen Produktionsstätten für Wäsche und Damenoberbekleidung angesiedelt hatten. Auch der Bereich Nordrhein war von der Textilindustrie geprägt - so nannte sich Krefeld zu recht die »Seidenweberstadt «.

Im Süden Baden-Württembergs gab es unzählige Textilunternehmen und mit Triumph und Schiesser die großen Weltunternehmen der Wäschebranche. Beide waren ursprünglich mehrstufige Unternehmen, die die zu verarbeitenden Stoffe selbst strickten und wirkten. Heute gilt dies nur noch für die Hersteller Mey und Trigema. Außerdem entwickelte sich von Oberfranken bis ins Fichtelgebirge eine starke Textilindustrie und im bayerischen Schwaben profitierte Augsburg von der aus Schlesien stammenden Dierig-Dynastie.

Die Bekleidungsindustrie entwickelte sich etwas später. An ihren Schwerpunkten in Ostwestfalen, Unterfranken und Berlin fehlten im Nachkriegsdeutschland bald Arbeitskräfte, so dass die Unternehmen mit kleineren Produktionsstätten in ländliche Regionen auswichen. Die Anzahl der Bekleidungsbetriebe wuchs von Mitte der 1950er Jahre bis Mitte der 1960er Jahre um rund 60 Prozent, was nahezu 2.000 Neugründungen entsprach.

Noch im Jahr 1995 lag der textile Schwerpunkt mit über 400 Betrieben und 47.000 Beschäftigten in Nordrhein-Westfalen, gefolgt von Bayern (285 Betriebe) und Baden-Württemberg (309 Betriebe) mit jeweils ca. 30.000 Beschäftigten. In den 167 sächsischen Betrieben waren zur gleichen Zeit 12.500 Arbeitnehmer:innen beschäftigt. In den übrigen Bundesländern war die Zahl der Textilbetriebe nur 
zweistellig oder noch geringer. ${ }^{4}$ In der DDR hatte die textile Be- und Verarbeitung ihren Schwerpunkt in Sachsen, wo in der Region Chemnitz, in der Lausitz und im Vogtland bis heute noch einige Betriebe bestehen.

\subsubsection{Die "Branche der armen Leute»}

Über 30.000 Jahre hinweg waren Spinnen und Weben handwerkliche Tätigkeiten, die im Gegensatz zu anderen Zünften nur wenig Wohlstand brachten. Im auslaufenden 18. Jahrhundert begann in der Textilindustrie die industrielle Revolution, die die Arbeitswelt der Menschen gravierend änderte. Mechanische Spinnmaschinen und Webstühle wurden durch Dampfmaschinen angetrieben, Textilien waren durch das neue Produktionssystem »Fabrik« maschinell und damit viel schneller und in größeren Mengen herstellbar.

Die bis dahin meist in Wohnhäusern in Handarbeit hergestellten Textilien verloren ihre Wettbewerbsfähigkeit. Die erwerbslos gewordenen Handwerker wanderten in die neu gegründeten Fabriken, aber ihre Hoffnung, so dem Elend zu entkommen, erfüllte sich nicht. Das 19. Jahrhundert war durch einen Unterbietungswettbewerb zwischen den Arbeitssuchenden gekennzeichnet, weil die maschinell erreichte höhere Produktivität zu einem Überangebot an Arbeitskräften führte. Die niedrigen Löhne in den Textilfabriken reichten nicht zum Überleben der Familien, so dass Frauen und oft auch Kinder mitarbeiten mussten. Sechs Tage die Woche schufteten sie 10 bis 14 Stunden täglich, um den Wohlstand der Textilfabrikanten zu mehren.

1854 erhielten Textilarbeiter nur 85 Prozent des durchschnittlichen Einkommens von Industriebeschäftigten und bis 1939 sank dieser Wert sogar auf 68 Prozent. ${ }^{5}$ Auch in der jungen Bundesrepublik gelang es nicht, diese Lücke zu schließen. Während andere Industriebranchen die Vorzüge der Mechanisierung nutzten und die Produktivität durch stetigen Ausbau erhöhten, waren viele Textilunternehmer weniger vorausschauend und verweigerten den dafür nötigen Kapitaleinsatz. Billige Arbeitskräfte und ein überalterter Maschinenpark blieben das Charakteristikum der Textilbranche. Die Gründe waren in der Nachkriegszeit dieselben wie zu Beginn der Industrialisierung: die traditionell geringgeachtete und schlechter bezahlte Frauenarbeit sowie vermeintlich einfache Tätigkeiten, die in den Augen der Arbeitgeber keine hohe Entlohnung rechtfertigten.

Der Anteil der Arbeiterinnen in der Bekleidungsindustrie betrug in den 1970er Jahren rund 85 Prozent und steigerte sich 1990 mit dem Strukturwandel von weniger Produktion zu mehr Logistiktätigkeit auf rund 88 Prozent. Das Beschäftigungsverhältnis in der Textilindustrie war aufgrund der Mechanisierung

4 Krippendorf/Holst/Richter (2009): Branchenanalyse Textilindustrie, S. 7.

5 Wassermann (1985): Arbeitsgestaltung als Gegenstand gewerkschaftlicher Politik, S. 68. 
deutlich ausgewogener: 1970 waren dort 54 Prozent Frauen beschäftigt, 1990 sank ihr Anteil auf 43 Prozent. ${ }^{6}$ Im Jahr 1954 waren 40 Prozent der weiblichen Beschäftigten ungelernt, weitere 40 Prozent gelernt oder angelernt und die restlichen 20 Prozent hatten zwar eine Ausbildung, übten aber einen anderen als den erlernten Beruf aus. ${ }^{7}$

Fest steht, dass sich die Textil- und die Bekleidungsindustrie wie kein anderer Industriezweig das traditionelle Frauenbild zunutze machte, wonach Frauenarbeit lediglich als Zuverdienst galt, weniger geachtet war und schlechter vergütet wurde. So waren die Textil- und die Bekleidungsbranche die »Arme-Leute-Industrie« - ein Zustand, der bis heute nachwirkt.

\subsubsection{Wirtschaftliche Entwicklung der Branchen}

Im Gegensatz $\mathrm{zu}$ anderen Industriebranchen in Deutschland mussten sich die Textil- und die Bekleidungsindustrie schon früh und fast gleichzeitig vier großen Problemen stellen:

- Die Abhängigkeit von importierten Rohstoffen und die damit verbundenen Währungsrisiken belasteten die Textilindustrie.

- Außerdem unterschätzen die Unternehmen das Abflauen der »Bekleidungswelle« in der Nachkriegszeit und standen so vor einer Überproduktion, die bereits 1958/1959 zu Kurzarbeit und Entlassungen führte.

- Die Wertigkeit von Kleidung in der gesellschaftlichen Wahrnehmung veränderte sich und andere Konsumgüter eroberten die privaten Haushalte - ein Trend, den die Bekleidungs- und die Textilbranche bis heute nicht umkehren konnten.

- $\mathrm{Zu}$ guter Letzt wurde diese Entwicklung durch eine Handelspolitik forciert, die weltweiten Warenströmen nur wenig Einhalt gebot. Auch der Versuch der Industrieländer, die Flut von Billigtextilien auf dem Weltmarkt durch die Importquoten verschiedener Welttextilabkommen einzuschränken, misslang (siehe Kapitel 2.1.5).

Bald nahmen die Arbeitsplatzverluste in der westdeutschen Textil- und Bekleidungsindustrie dramatische Ausmaße an: 1957 war mit 965.000 Beschäftigten der Höchststand in den textilen Branchen erreicht. Bis 1975 ging ein Drittel dieser Arbeitsplätze verloren; 1985 waren es mit 420.000 Arbeitsplätzen nicht einmal mehr die Hälfte. Nach der deutschen Wiedervereinigung stabilisierte sich die

6 Statistisches Bundesamt (1970): Reihe 15, S. 13; Statistisches Bundesamt (1990): Fachserie 16, Reihe 2.1, S. 16.

7 GTB: Geschäftsbericht 1953-1954 des Hauptvorstandes, S. 198. 
Zahl zwar kurzfristig mit rund 371.000 Beschäftigten in 1990 (siehe Abbildung 3), aber danach setzte sich der Strukturwandel unerbittlich fort und erfasste auch die meisten ostdeutschen Betriebe mit voller Wucht. Bis zum Jahr 2000 halbierte sich die Zahl der bundesweit Beschäftigten auf rund 185.000.

Abbildung 3: Strukturdaten im Textil-und Bekleidungsgewerbe (1951-2000)

\begin{tabular}{|l|l|l|l|l|l|l|}
\hline & 1951 & 1960 & 1970 & 1980 & $1990^{*}$ & $2000^{*}$ \\
\hline Betriebe ${ }^{1)}$ & 7.389 & 8.999 & 7.704 & 5.392 & 3.526 & 1.873 \\
\hline Beschäftigte ${ }^{1)}$ & 809.506 & 947.433 & 869.911 & 541.232 & 370.666 & 185.195 \\
\hline $\begin{array}{l}\text { Anteil am Verarbeitenden } \\
\text { Gewerbe }\end{array}$ & $17,1 \%$ & $12,7 \%$ & $10,1 \%$ & $7,4 \%$ & $5,1 \%$ & $3,0 \%$ \\
\hline $\begin{array}{l}\text { Bruttolohn- und gehalts- } \\
\text { summe } \\
\text { (in Mio. Euro) }\end{array}$ & - & 2.366 & 4.885 & 6.554 & 6.745 & 4.693 \\
\hline Lohnquote & - & $19,9 \%$ & $23,8 \%$ & $23,9 \%$ & $19,4 \%$ & $17,8 \%$ \\
\hline $\begin{array}{l}\text { Umsatz } \\
\text { (in Mio. Euro) }\end{array}$ & 8.277 & 11.871 & 20.533 & 27.476 & 34.825 & 26.409 \\
\hline $\begin{array}{l}\text { Anteil am Verarbeitenden } \\
\text { Gewerbe }\end{array}$ & $15,5 \%$ & $9,1 \%$ & $7,3 \%$ & $4,6 \%$ & $3,8 \%$ & $2,0 \%$ \\
\hline $\begin{array}{l}\text { Auslandsumsatz } \\
\text { (in Mio. Euro) }\end{array}$ & 391 & 797 & 2.059 & 4.610 & 8.837 & 8.594 \\
\hline Exportquote & $4,7 \%$ & $6,7 \%$ & $10,0 \%$ & $16,8 \%$ & $25,4 \%$ & $32,5 \%$ \\
\hline $\begin{array}{l}\text { Produktion }{ }^{2)} \\
\text { (in Mio. Euro) }\end{array}$ & - & - & 24.454 & 24.769 & 26.081 & 16.375 \\
\hline $\begin{array}{l}\text { Einfuhren }{ }^{2)} \\
\text { (in Mio. Euro) }\end{array}$ & - & - & 4.504 & 14.239 & 25.646 & 31.730 \\
\hline $\begin{array}{l}\text { Ausfuhren }{ }^{2)} \\
\text { (in Mio. Euro) }\end{array}$ & - & - & 2.774 & 8.572 & 16.519 & 18.943 \\
\hline $\begin{array}{l}\text { Inlandsmarkt } \\
\text { (in Mio. Euro) }\end{array}$ & - & 26.184 & 30.436 & 35.208 & 29.162 \\
\hline $\begin{array}{l}\text { Einfuhrüberschuss } \\
\text { (in Mio. Euro) }\end{array}$ & - & 1.730 & 5.668 & 9.127 & 12.787 \\
\hline
\end{tabular}

* Berichtskreis: bis 1980 früheres Bundesgebiet, ab 1990 Gesamtdeutschland

1) nach Betrieben (WZ-2008-Code 13, 14)

2) nach Güterklassen und Güterabteilungen (GP-2009-Code 13, 14)

Eigene Darstellung; Datenquellen: Statistisches Bundesamt (Destatis); Bundesamt für Wirtschaft und Ausfuhrkontrolle (BAFA); eigene Berechnungen. 
Im Gegensatz zur rückläufigen Inlandsproduktion nahm der textile Außenhandel im Laufe der Jahre an Fahrt auf. Bekleidung war ein - im wahrsten Sinne des Wortes - leicht zu handelndes Gut und die Herstellung konnte überwiegend mit menschlicher Arbeitskraft bewerkstelligt werden. Solange billige Arbeitskräfte zur Verfügung standen, erschien den Textilunternehmern die Investition in teure Maschinen eher zweitrangig. Unterstützt wurde diese Einstellung durch eine entsprechende Außenhandelspolitik, die wieder eingeführte Waren gänzlich oder teilweise von Abgaben befreite. Die sogenannte passive Lohnveredelung ermöglichte es den Unternehmen, die Produktion ins billigere europäische Ausland $\mathrm{zu}$ verlagern und die Ware zur Fertigstellung wieder zu importieren. Allein 1970 betrug der Beschäftigungsanteil der osteuropäischen Länder an der weltweiten Textil- und Bekleidungsindustrie 19 Prozent, der Anteil der Entwicklungsländer lag sogar bei 51 Prozent. ${ }^{8}$

Die Möglichkeit der passiven Lohnveredelung und die im Ausland gefertigte Billigkleidung ließ die Textilimporte rasant steigen. Wurden 1970 noch Textilien im Wert von 4,5 Milliarden Euro importiert, hatte sich dieser Wert 1980 bereits verdreifacht und 1990 um weitere 80 Prozent auf fast 25,6 Milliarden Euro erhöht. Nach Berechnungen des Instituts der Deutschen Wirtschaft stieg der Anteil der deutschen Textil- und Bekleidungsimporte an der Inlandsversorgung von 19 Prozent im Jahr 1970 auf 80 Prozent zur Jahrtausendwende. ${ }^{9}$ Die größten Vorleistungsproduzenten waren Bekleidungsunternehmen in Polen, Rumänien und Tunesien mit einem Anteil von ca. 40 Prozent, weitere 20 Prozent der Produkte wurden in Tschechien, der Slowakei, Kroatien und Ungarn bearbeitet. ${ }^{10}$

Obwohl die inländische Produktion von Textilien und Bekleidung abnahm, vervielfachte sich die Zahl der Ausfuhren in jedem Jahrzehnt. Die Exportquote, die 1951 noch 4,7 Prozent betrug, konnte bis 1970 verdoppelt werden (siehe Abbildung 3). 1980 wurden Waren im Wert von rund 8,6 Milliarden Euro ausgeführt, was einem Exportanteil von 16,8 Prozent entsprach; 1990 wurden bereits 25,4 Prozent aller textilen Produkte exportiert. Da die Bekleidungshersteller die Vorteile der passiven Lohnveredelung früh nutzten und der überwiegende Teil der in Deutschland verkauften Bekleidung bald billig im Ausland hergestellt wurde, verzeichneten die Textil- und die Bekleidungsindustrie seit der Nachkriegszeit immer einen Einfuhrüberschuss. 1970 lag er bei 1,7 Milliarden Euro und hatte sich

8 Fröbel/Heinrichs/Kreye (1977): Die neue internationale Arbeitsteilung, S. 72.

9 Zum Vergleich: Der Anteil aller ausländischen Produkte am gesamten deutschen Warenhandel betrug im Jahr 2000 nur 40,5 Prozent; vgl. Grömling/Matthes (2003): Globalisierung und Strukturwandel der deutschen Textil- und Bekleidungsindustrie, S. 51.

10 Grömling/Matthes (2003): Globalisierung und Strukturwandel der deutschen Textil- und Bekleidungsindustrie, S. $47 \mathrm{ff}$. 
bis 1990 auf 9,1 Milliarden Euro verfünffacht, während der Branchenumsatz im selben Zeitraum um rund 70 Prozent stieg.

Mit dem Rückgang der Branchen nahm im Laufe der Jahre auch der textile Anteil an der allgemeinen Wertschöpfung ab. 1951 machte der textile Branchenumsatz mit 8,3 Milliarden Euro noch 15,5 Prozent der gesamtwirtschaftlichen Erlöse aus. 1970 hatte sich der Anteil auf 7,3 Prozent halbiert und nach der Wiedervereinigung sank er auf 3,8 Prozent (siehe Abbildung 3). Im Laufe dieser Entwicklung hatten die Textil- und die Bekleidungsindustrie ihr anfängliches Wirtschaftsgewicht als bedeutende Konsumgüterbranche eingebüßt.

\section{Die 1950er Jahre: Stotternd durch das Wirtschaftswunder}

Die »Wirtschaftswunderjahre« der jungen Bundesrepublik waren geprägt von hohen Wachstumsraten, einer sinkenden Arbeitslosenquote (von 11 Prozent in 1950 auf 1,3 Prozent in 1960), steigenden Realeinkommen und damit einer spürbaren Verbesserung der aktuellen Lebenssituation breiter Bevölkerungsschichten. Die Nettorealeinkommen der Beschäftigten stiegen in diesem Jahrzehnt um gut zwei Drittel. ${ }^{11}$

1950 arbeiteten noch 597.000 Menschen in der Textil- und 213.000 in der Bekleidungsindustrie, um den steigenden Bedarf an Kleidung zu stillen. ${ }^{12}$ Trotzdem wurde schon in den ersten Jahren des Aufschwungs die starke Volatilität sichtbar, der sich die Textil- und die Bekleidungsindustrie wie keine andere Branche anpassen mussten. Während die Gesamtwirtschaft 1952 das zweite Jahr in Folge ein Wachstum von über 9 Prozent verzeichnete, durchlebte die Textilindustrie ihre erste Rezession. Der Umsatz der Textilhersteller brach um 13 Prozent ein. Ursache war der Koreakrieg, der die Rohstoffpreise kurzfristig in die Höhe schießen ließ, so dass die Textilunternehmen auf ihren teuer gefüllten Lagern sitzenblieben. Diese erste kurze Krise kostete die Branche 30.000 Arbeitsplätze, wobei der Abschwung die Bekleidungsindustrie weniger hart traf. 1952 gelang ihr noch ein schwaches einprozentiges Umsatzwachstum.

Nach der Koreakrise nahmen die Textil- und die Bekleidungsindustrie wieder am Aufschwung der 1950er Jahre teil. Die Wachstumsraten waren beachtlich, blieben aber hinter der gesamtwirtschaftlichen Entwicklung zurück. Von 1950 bis 1959 verdoppelte sich die westdeutsche Wirtschaftsleistung, ${ }^{13}$ während die Um-

11 Bispinck u. a. (2019): 70 Jahre Tarifvertragsgesetz, S. 4.

12 Quelle für alle Branchendaten der Textil- und der Bekleidungsindustrie hier und im Folgenden, falls nicht anders vermerkt: Statistisches Bundesamt (Destatis) (2008): Fachserie 4, Reihe 4.1.1 (siehe Tabelle 8 und 9 im Anhang).

13 Statistisches Bundesamt (Destatis) (2021): Volkswirtschaftliche Gesamtrechnungen. Bruttoinlandsprodukt, Bruttonationaleinkommen, Volkseinkommen. Lange Reihen ab 1925. 
satzzuwächse der Textilindustrie nur um 65 Prozent und die der Bekleidungsindustrie um 79 Prozent stiegen.

Schon 1957/1958 kam es zum nächsten Einbruch: Die sogenannte Textilkrise traf die Unternehmen hart. 1957 erreichte die Zahl der in der Textilindustrie Beschäftigten mit 648.000 ihrem Höhepunkt, ${ }^{14}$ aber schon im Folgejahr wurden 29.000 Textilbeschäftige entlassen. Auch wenn die Bekleidungsindustrie als Hauptabnehmer in den Sog der Textilkrise geriet, fiel der Beschäftigungsabbau von fast 3.500 Arbeitsplätzen im Jahr 1958 vergleichsweise gering aus. Deutlich härter traf es z. B. die Kohlereviere: Durch die tiefe Krise im Kohlebergbau verloren von 1956 bis 1960 fast 77.000 Kumpel ihren Arbeitsplatz. ${ }^{15}$

Zusätzlich zum Beschäftigtenabbau wurde in den Textilbetrieben wie auch in den Kohlezechen kurzgearbeitet. Während der Kohlebergbau jedoch von der Bundesregierung und den betroffenen Landesregierungen stark subventioniert wurde, gelang es der Textilindustrie aufgrund ihrer mittelständischen Organisationsstruktur nicht, Unterstützungen in ähnlichem Umfang durchzusetzen. Ein vergleichbarer politischer Druck gelang nicht, weil Entlassungen und Verdienstminderungen beim Niedergang von Textilbetrieben geografisch stärker verteilt waren und zudem deutlich weniger Menschen pro Betrieb vom Arbeitsplatzabbau betroffen waren als bei einer Zechenschließung.

Ausgelöst wurde die Textilkrise durch Überproduktion, zudem wurde mit einem veralteten Maschinenpark zu teuer produziert. Parallel zur Vernachlässigung ihrer Anlagen mussten sich die Hersteller mit neu aufkommenden Produkten aus Kunststoff und synthetischen Fasern auseinandersetzen. Außerdem hatten große Teile der Branche den Wandel vom bisher gewohnten Verkäufermarkt zu einem Käufermarkt schlicht verschlafen, weil sie immer noch den Nachholbedarf der Nachkriegszeit vor Augen hatten.

Als Folge investierte die Branche in moderne Anlagen, um kostengünstiger produzieren zu können. Die dadurch erzielten Produktivitätssteigerungen und die damit verbundenen Personalreduzierungen brachten den Unternehmen erhebliche Kostenvorteile. Dies betraf insbesondere die Webereien, wo es zu ganz erheblichen Rationalisierungen kam. Die GTB-Verwaltungsstelle Jöllenbeck (Kreis Bielefeld) beschrieb z. B. die Auswirkungen in der Futterstoffweberei Delius \& Söhne in Spenge: Im Jahr 1950 bedienten 250 Weber:innen insgesamt 520 Webmaschinen. Da doppelschichtig gearbeitet wurde, fanden 500 Menschen Beschäftigung. Im Jahr 1959, nach Einführung der Webautomaten, arbeiteten nur noch 8 Weber:innen an 500 Webautomaten. Für den Dreischichtbetrieb waren so-

14 Zum Vergleich: In der Bekleidungsindustrie wurde erst 1966 mit 408.077 Beschäftigten der Höchststand erreicht, bevor es zur Trendwende bei den Arbeitsplätzen kam.

15 Wikipedia-Artikel »Ruhrbergbau«, Abschnitt Geschichte« (Abruf am 16.1.2021). 
mit nur noch 24 Weber:innen nötig. 1960 hatte sich der Anteil der Webautomaten in der Branche gegenüber 1950 vervierfacht und machte nun über 50 Prozent aus. ${ }^{16}$

Ende der 1950er Jahre hatte die Branche mit einer weiteren Herausforderung zu kämpfen: Die Textilimporte übertrafen die textilen Exporte. Neben der Konkurrenz des traditionell sehr starken Textillandes Italien drängten auch japanische Wettbewerber auf den Textilmarkt. ${ }^{17}$ Als protektionistische Maßnahme für die Textilindustrie setzte Bundeswirtschaftsminister Ludwig Erhard auf der GATT-Konferenz 1959 eine erste dreijährige »Schonfrist« gegenüber der ausländischen Konkurrenz durch. In dieser Zeit sollte die Branche ihre Anlagen und Organisationsstrukturen modernisieren und auch rationalisieren, um auf dem internationalen Markt wettbewerbsfähig zu werden. Zur Unterstützung sollten die Unternehmen Kredite aus dem »European Recovery Program« (ERP) beantragen, was sie im Volumen von 20 Millionen DM auch taten. ${ }^{18}$

\section{Die 1960er Jahre: Der Wettbewerbsdruck nimmt zu}

In den 1960er Jahren stieg das Bruttosozialprodukt in der Bundesrepublik jährlich zwischen 5 und 6 Prozent; die Arbeitslosigkeit sank von 1,3 Prozent in 1960 auf 0,7 Prozent in 1966. Die Nettorealeinkommen stiegen in diesem Jahrzehnt um 30 Prozent. ${ }^{19}$ In der Textilindustrie verstärkte sich in den 1960er Jahren der internationale Wettbewerbsdruck. Hersteller klassischer Textilprodukte wie Standardgarne oder einfacher Stoffe gerieten unter Druck.

Die Unternehmer reagierten auf diese Entwicklung recht unterschiedlich. Die einen gaben die nicht mehr wettbewerbsfähige Herstellung von Stapelware auf und stiegen auf Schaft- und Jacquardwaren ${ }^{20}$ um; andere blieben untätig und hofften letztlich darauf, dass genügend inländische Konkurrenten die Produktion umstellen und dadurch Überkapazitäten abgebaut würden. Die dritte Gruppe sah das Heil in hoch technisierter Massenherstellung, mit der man kostengünstig produzieren und die ausländische Konkurrenz in Schach halten konnte. Die Produktionsanlagen wurden stärker automatisiert und die Webtechnik erlebte durch Projektilwebautomaten ${ }^{21}$ einen Produktivitätssprung um das Fünf-

16 CTB: Bericht der Verwaltungsstelle Jöllenbeck an den Hauptvorstand, undatiert (1962), AdsD $5 /$ GTBA410212.

17 Lindner (2001): Den Faden verloren, S. 112.

18 Lindner (2001): Den Faden verloren, S. 114.

19 Bispinck u. a. (2019): 70 Jahre Tarifvertragsgesetz, S. 5.

20 Jacquard ist eine von Joseph-Marie Jacquard (1752-1834) erfundene Bindungsart für Stoffe, die eine Gestaltung mit komplexen Mustern ermöglicht. Verschiedene Materialien wie etwa Seide, Viskose, Baumwolle oder synthetische Stoffe können so gewebt werden und ergeben z. B. Brokat oder Damast.

21 Nicht mehr das »Weberschiffchen« mit der Garnspule wurde durch die Kettfäden, also die Längsfäden des Cewebes geführt, stattdessen nahm ein wenige Cramm schweres Projektil 
fache. ${ }^{22}$ Die Mehrheit aller Textilfabrikanten sah jedoch die Bundesregierung in der Pflicht und erwartete deren »rettenden Eingriff« vor allem durch protektionistische Maßnahmen.

Im Jahrzehnt des "Wirtschaftswunders« kam ein weiteres Problem auf die textilen Mittelständler zu: Beschäftigte, denen es fachlich und geografisch möglich war, wanderten in besser bezahlte Industriebranchen ab. Die Textil- und die Bekleidungsindustrie galten in der Bevölkerung nach wie vor als Krisenbranchen mit niedrigen Löhnen, verbreiteter Kurzarbeit und harten Arbeitsbedingungen wie Schichtarbeit, Lärm und Staub. So ging den Branchen neben der Arbeitskraft auch viel Fachwissen unwiederbringlich verloren.

Viele Bekleidungshersteller wichen nun in ländliche Regionen aus, wo insbesondere weibliche Arbeitskräfte mangels alternativer Arbeitsplätze wohnortnah und zu geringerem Lohn beschäftigt werden konnten. Verglichen mit großen Anlagen waren Nähmaschinen leicht $\mathrm{zu}$ transportieren und wegen deren geringen Gewichts benötigten die Unternehmen keine besondere fabrikmäßige Infrastruktur. Außerdem warben die Textil- und Bekleidungshersteller wie auch die großen Industriebranchen in der Zeit der Vollbeschäftigung um »Gastarbeiter:innen« aus süd- und südosteuropäischen Ländern. Im bundesweiten Industrievergleich war der Anteil der Beschäftigten mit Migrationshintergrund in den Textilbranchen mit 17 bis 20 Prozent am höchsten. ${ }^{23}$

Ein weiterer Vorteil einer Produktion jenseits der Ballungsgebiete war, dass die dortigen Unternehmen staatliche Fördergelder erhielten, um die Industrialisierung in ländlichen strukturschwachen Gebieten wie dem Bayerischen Wald oder der Oberpfalz und in sogenannten Zonenrandgebieten voranzutreiben. Eine Mitte der 1980er Jahre veröffentlichte Studie schrieb vor allem den Unternehmen der Bekleidungsindustrie den "größten Nutzen aus der regionalpolitischen Förderung « zu. ${ }^{24}$ Beispielsweise profitierte der Miederwarenhersteller Triumph mit seinen neuen Betriebsstätten »auf dem Lande« in Wegscheid, Kötzting, Tittling, Landau/Isar oder Donauwörth von dieser Maßnahme.

Insbesondere in der ersten Hälfte der 1960er Jahre legte die Entwicklung der Textil- und der Bekleidungsindustrie damit ein Tempo vor, das viele andere Wirtschaftsbereiche überflügelte. Der Umsatz von etwa 2 Milliarden Euro im Jahr 1954 konnte bis Mitte der 1960er Jahre verdreifacht werden. 1966, als die Beklei-

den Faden auf und führte ihn durch die Kette. Der Webvorgang konnte so wesentlich schneller durchgeführt werden. In den 1960er und 1970er Jahren wurde diese Technik erweitert, indem die Schussfäden von Wasser- oder Luftdüsen vorangetragen wurden.

22 Lindner (2001): Den Faden verloren, S. 68.

23 CTB: Ceschäftsberichte des Hauptvorstandes.

24 Engel, Jürgen: Internationale Wirtschaftsbeziehungen und Strukturwandel am Beispiel der bundesdeutschen Textil- und Bekleidungsindustrie, Bremen 1985, S. 140; zit. nach Lindner (2001): Den Faden verloren, S. 141. 
dungsindustrie mit über 5.600 Betrieben die meisten Produktionsstätten ihrer Geschichte hatte, setzte eine gesamtwirtschaftliche Rezession ein, die die Textilund die Bekleidungsindustrie besonders schwer traf. Diese Sonderrolle der überproportional negativen Betroffenheit sollte sie auch in kommenden Krisen nicht mehr loswerden. Während das Bruttoinlandsprodukt im Rezessionsjahr 1967 um 0,3 Prozent zurückging, ${ }^{25}$ brachen die Umsätze der Textilindustrie um 7 Prozent und die der Bekleidungsindustrie sogar um 9 Prozent ein. Die Unternehmen reagierten mit einem drastischen Arbeitsplatzabbau und entließen in beiden Branchen 9 Prozent der Beschäftigten.

Nach dem Scheitern der Regierung von CDU/CSU und FDP unter Bundeskanzler Ludwig Erhard im Jahr 1966 implementierte der neue sozialdemokratische Wirtschaftsminister Karl Schiller als Reaktion auf die erste gesamtwirtschaftliche Rezession in der Bundesrepublik ein Konzept der gesamtwirtschaftlichen Globalsteuerung. Bald boomte die westdeutsche Wirtschaft wieder und auch in der Textil- und der Bekleidungsindustrie ging es bergauf. Von 1968 bis 1972 erhöhte sich die Wirtschaftsleistung um 21 Prozent, die Umsätze der Textilindustrie stiegen um 25 Prozent und die der Bekleidungsindustrie sogar um 45 Prozent.

Die breitere Verwendung von Textilien ließ den weltweiten Pro-Kopf-Verbrauch in den Industrieländern von 1960 bis 1970 um 25 Prozent steigen, weltweit stieg er von 1950 bis 1970 um ein Drittel. ${ }^{26}$ Der zunehmende Wohlstand erhöhte neben dem Bedarf an Kleidung auch den Bedarf an Heimtextilien wie z. B. Vorhängen, Möbelstoffen oder Teppichen. Die wachsende Automobilindustrie benötigte ebenfalls Gewebe für Autositze und Textilien für die Innenverkleidung. Mit den technischen Textilien entstand ein neuer Verwendungszweck. Ihre vielfältigen Anwendungsbereiche beispielsweise als Dämmmaterialien, Autogurte, Windeln oder im Medizinbereich erforderten neue textile Eigenschaften. Später entwickelten sich weitere Nutzungen in Sport und Transport, beim Einsatz in Hoch- und Tiefbau und beim Flugzeugbau.

Aufgrund der Rationalisierungsmaßnahmen konnten die Textilbeschäftigten jedoch nicht vom Aufschwung der Jahre 1968 bis 1972 profitieren. In dieser Zeit büßte die Branche 29.500 Arbeitsplätze ein. Die neuen Technologien bei der Flächenproduktion von Textilien erhöhten die Produktivität zwar erheblich, verlangten zugleich aber auch einen hohen Kapitaleinsatz. Erfolgreich waren nur die Unternehmen, die den Markt mit Massenprodukten belieferten und ihre Produktpalette ausweiteten. Anders sah es in der Schwesterbranche aus: Die weniger mechanisierte Bekleidungsindustrie stellte in diesen vier Jahren 3.500 Näher:innen ein.

25 Statistisches Bundesamt (Destatis) (2021): Volkswirtschaftliche Gesamtrechnungen. Bruttoinlandsprodukt, Bruttonationaleinkommen, Volkseinkommen. Lange Reihen ab 1925.

26 Vgl. Lindner (2001): Den Faden verloren, S. 54. 


\section{Die 1970er Jahre: Rezession, Struktur- und Kulturwandel treffen aufeinander}

Die Branchenprobleme aufgrund stetig wachsender Textil- und Bekleidungsimporte sowie dem Wandel im Verbraucherverhalten wurden durch den Ölpreisschock Ende 1973 und die darauffolgende Rezession verstärkt. Die Strategie der Massenproduktion beförderte die Bildung starker Konzerne: Konkurrenten, wenn auch zum Teil sanierungsbedürftig, wurden übernommen und auf großindustrielle Massenfertigung umgestellt, Basisartikel wurden in großen Serien hergestellt und der Abnehmerkreis auf möglichst große Kunden konzentriert. Genau diese Strategie der Massenproduktion und der darauf ausgerichtete Maschinenpark nahmen den Unternehmen aber die Möglichkeit, flexibel auf Kundenwünsche zu reagieren.

Mit der Wirtschaftskrise 1974 sank die Nachfrage. Vor allem bei den relativ unbeweglichen Großunternehmen kam es in der Folge zu spektakulären Firmenzusammenbrüchen. So waren 1976 von der Pleite der bayerischen Glöggler-Textilgruppe 12.500 Arbeitnehmer:innen betroffen. ${ }^{27}$ Ein weiteres prominentes Beispiel ist das Textilunternehmen van Delden mit ehemals 7.000 Beschäftigten, das 1973 noch stark expandierte und 1980 spektakulär in die Pleite ging. ${ }^{28}$ Von 1970 bis 1975 halbierte sich der Marktanteil von Schulte \& Dieckhoff, dem führenden Produzenten von Maschenware, der überwiegend niedrigpreisige Nahtlosfeinstrümpfe herstellte. ${ }^{29}$ Aber auch viele kleine und mittlere Unternehmen verschuldeten sich aufgrund des hohen Kapitaleinsatzes und verschwanden vom Markt, weil die Umsätze wegbrachen oder weniger ertragreich wurden. Wenig förderlich war in dieser Situation die Stabilitätspolitik der Bundesregierung mit restriktiver Kreditvergabe und hohen Schuldzinsen. ${ }^{30}$

In dieser Zeit gelang es nur wenigen Textilunternehmen, sich mit qualitativ hochwertigen oder innovativen Produkten am Weltmarkt zu behaupten. Der heimische Branchenvorteil lag laut einer Studie in der »vermutlich höheren Flexibilität kleinerer Unternehmen, die gerade durch Änderungen der Verbrauchsnachfrage Mitte der siebziger Jahre besonders wichtig wurde «. ${ }^{31}$ Kleinere Betriebsoder Unternehmenseinheiten konnten deutlich schneller auf Kundenwünsche reagieren als große Unternehmen, vorausgesetzt sie behielten den aktuellen Überblick über das globale Marktgeschehen. Doch aller Innovation zum Trotz

27 Pfleger-Edel (1976): Der Fall Glöggler hat Textilindustrie psychologischen Schock versetzt, in: Handelsblatt vom 26.3.1976.

28 Wassermann (1985): Arbeitsgestaltung als Gegenstand gewerkschaftlicher Politik, S. 75.

29 Lindner (2001): Den Faden verloren, S. 155.

$30 \mathrm{Vgl}$. Lindner (2001): Den Faden verloren, S. 151.

31 Hergeth, Helmut: Investitionsstrategien für stagnierende Branchen: dargestellt am Beispiel der Textilindustrie der Bundesrepublik Deutschland, Münster 1986, S. 15f.; zit. nach Lindner (2001): Den Faden verloren, S. 158. 
blieb das Branchenwachstum in den folgenden Jahrzehnten immer weiter hinter dem der anderen Industriezweige zurück.

Die kapitalintensive Produktion der Textilindustrie erfordert längere Maschinenlaufzeiten, die am vorteilhaftesten durch Schichtarbeit möglichst an sieben Tagen pro Woche umgesetzt wurden. In den Niedriglohnländer galten keine oder nur geringe Beschränkungen bei Schicht-, Nacht- und Feiertagsarbeit und es gab eine große »Reservearmee« von Arbeiter:innen, die notgedrungen zu jeder Zeit arbeiten mussten. Aus kapitalistischer Sicht waren dies ideale Voraussetzungen für die Verlagerung auch teurer Produktionsanlagen. ${ }^{32} 1977$ analysierten Wissenschaftler des Max-Planck-Instituts zur Erforschung der Lebensbedingungen der wissenschaftlich-technischen Welt ${ }^{33}$, dass die deutsche Textil- und Bekleidungsbranche ihre Marktposition »wohl kaum« durch Kreativität, Schnelligkeit und Pünktlichkeit bei der Lieferung sowie hoher Qualität behaupten könne. Für Fertigungen mit solchen Attributen sei der Markt begrenzt und die Niedriglohnländer würden auf allen Gebieten, gerade unter Leitung und Mitwirkung bundesdeutscher Unternehmen, aufholen. ${ }^{34}$

Im Gegensatz zur Textilindustrie gab es in der Bekleidungsindustrie kaum Rationalisierungsmöglichkeiten. Die arbeitsteilige Fertigung in der Massenproduktion konnte die handwerklichen Tätigkeiten nicht gänzlich ersetzen. Roboter schweißten feste Autoteile schnell zusammen, konnten aber sogenannte biegeschlaffe Materialien wie Stoffe nicht zusammenfügen. Diese instabilen Teile mussten - und müssen immer noch - von Hand und mittels Nähmaschine zu einem Stück verarbeitet werden. Die verstärkte Verlagerung dieser Arbeitsschritte in Länder mit Niedriglöhnen schien vielen Unternehmen als der Ausweg. Durch eine Mischkalkulation aus höheren inländischen und niedrigeren ausländischen Lohnkosten sollte die Ertragslage verbessert werden.

Die Ertragssteigerung war für die Bekleidungsunternehmen aber nur von kurzer Dauer, denn der Einzelhandel forderte umgehend die Weitergabe dieser Kostenersparnis und drohte damit, selbst im Ausland auf Einkaufstour zu gehen. Auch die Handelsbeschränkungen durch die Welttextilabkommen (siehe Kapitel 2.1.5) konnten nicht verhindern, dass immer größere Teile der Produktion erst ins europäische und später ins asiatische Ausland verlagert wurden. Ein Paradebeispiel ist der Miederhersteller Triumph:Anfang der 1960er Jahre arbeiteten noch 16.000 Beschäftigte an westdeutschen Standorten und »nur « 4.000 Beschäftigte im Ausland. Zwanzig Jahre später hatte sich das Verhältnis ins Gegenteil verkehrt:

32 Fröbel/Heinrichs/Kreye (1977): Die neue internationale Arbeitsteilung, S. 209.

33 Das in Starnberg ansässige Institut wurde 1970 gegründet und beschäftigte sich unter der Leitung von Carl Friedrich von Weizsäcker unter anderem mit Globalisierungsfragen. Nach internen Auseinandersetzungen wurde es 1984 geschlossen.

34 Fröbel/Heinrichs/Kreye (1977): Die neue internationale Arbeitsteilung, S. 211. 
Knapp 4.000 inländischen Beschäftigten standen nun 11.000 im Ausland gegenüber. ${ }^{35}$

\section{Die 1980er Jahre: „Tal der Tränen« durchschritten?}

Der nächste Schlag für die Textil- und die Bekleidungsindustrie war die scharfe Rezession, die 1982 ihren Höhepunkt erreichte. Zum ersten Mal in der Geschichte der Bundesrepublik gab es über 2 Millionen Arbeitslose; die sozialliberale Regierung wurde von der konservativ-liberalen Koalition abgelöst. Die politische Wende hin zu einer stark angebotsorientierten Wirtschafts- und Finanzpolitik hatte einen drastischen Abbau sozialer Leistungen und Schutzrechte zur Folge. Eine nachhaltige Beschäftigungspolitik lag nun nicht mehr im Fokus der Bundesregierung, so dass sich die Gewerkschaften für eine tarifvertragliche Arbeitszeitverkürzung einsetzten.

Von 1981 bis 1983 gingen in den textilen Branchen rund 78.400 Arbeitsplätze verloren. In der Bekleidungsindustrie war ein Rückgang um 24 Prozent, in der Textilindustrie um 22 Prozent zu verzeichnen. Von der knappen Million Arbeitsplätze in diesen Branchen Mitte der 1960er Jahre war nach knapp zwanzig Jahren nicht einmal mehr die Hälfte übrig. ${ }^{36}$

Als es im Zuge des Konjunkturaufschwungs Mitte der 1980er Jahre zu deutlichen Umsatzzuwächsen kam, gingen viele davon aus, man habe das »Tal der Tränen« durchschritten. Einige klassische Textilunternehmen wie Freudenberg und Hartmann hatten der Krise mit innovativen Produkten für technische und medizinische Anwendungen die Stirn geboten, mittelgroße Unternehmen hatten sich durch Spezialanfertigungen auch in klassischen Anwendungsbereichen behauptet. Der Einsatz modernster Technik und eine hohe Flexibilität trugen endlich Früchte. Der ununterbrochene Beschäftigungsabbau seit 1970 wurde als »Gesundschrumpfen " gewertet. $^{37}$

Im Rückblick muss festgestellt werden, dass diese Sichtweise zu optimistisch war. Der Textil- und der Bekleidungsindustrie sollte die Trendwende nicht gelingen. Die Schutzmaßnahmen des Welttextilabkommens begrenzten lediglich den Zuwachs ausländischer Produkte und streckten die Zeiträume, aber die strategische Abwanderung der Produktion verhinderten sie nicht. Die verlagerte Bekleidungsindustrie bezog ihre Vorprodukte immer weniger aus Deutschland; die Produktion technischer Textilien verlor ihr Alleinstellungsmerkmal und fand bald Nachahmer in aller Welt.

35 Wassermann (1985): Arbeitsgestaltung als Gegenstand gewerkschaftlicher Politik, S. 75.

36 Laut Statistischem Bundesamt (Destatis) gab es 1966 in der Textil- und der Bekleidungsindustrie 945.843 Beschäftigte, 1983 waren es nur noch 435.431 Beschäftigte; siehe Tabelle 8 und 9 im Anhang.

37 Lindner (2001): Den Faden verloren, S. 173. 


\section{Die 1990er Jahre: Die Wiedervereinigung als "textiles Strohfeuer"}

Die Lausitz und Sachsen und waren traditionelle Textilschwerpunkte in der DDR. 1989 waren in den großen, zu »volkseigenen« Textil- und Bekleidungskombinaten zusammengefassten Unternehmen rund 300.000 Menschen beschäftigt. Die DDR-Textilindustrie war die größte in den Ostblockstaaten und trotzdem durch ineffiziente Fertigungsabläufe und veraltete Maschinentechnik geprägt. Auch wenn sie modisch auf westlichem Stand bleiben wollte, brachte die Zentralisierung in den Verwaltungsebenen sie an ihre Grenzen. Zwar wurden in der DDR Textilmaschinen aufWeltniveau produziert, die moderne Technik fand ihren Weg in die örtlichen Textilfabriken jedoch erst mit zeitlicher Verzögerung. Export und Devisengeschäft hatten bei den Verantwortlichen eine höhere Priorität als die Versorgung der heimischen Industrie. So wurde vielfach bis 1990 mit Maschinen aus der Zeit vor dem Zweiten Weltkrieg produziert.

Während in ostdeutschen Geschäften kaum hochwertige Textilien aus eigener Fertigung zu kaufen waren, fand sich in westdeutschen Modekatalogen Kleidung aus Plauen und Dresden. Die Qualität war trotz meist antiquierter Technik auf hohem Niveau und das Billiglohnland DDR wurde zu einem wichtigen Versorger für den westdeutschen Markt. ${ }^{38}$ Mit der deutschen Wiedervereinigung wurde klar, dass dieser Erfolg auf den besonderen Verrechnungskursen von D-Mark und Mark der DDR beruhte. Ab Mitte 1990 galt für den gesamtdeutschen Binnenhandel nur noch das Verhältnis 1:1, das den unproduktiven Branchen den Todesstoß versetzte. Innerhalb von vier Jahren verschwand die ostdeutsche Bekleidungsindustrie nahezu komplett, die Zahl der Beschäftigten in der implodierten Textilindustrie schrumpfte auf nur noch ein Zehntel ihrer ursprünglichen Größe.

Den westdeutschen Textilunternehmen brachte die Wiedervereinigung einen kurzen kräftigen Aufschwung. Ihre Umsätze stiegen 1989/1990 um 11 Prozent und die Bekleidungshersteller konnten ihren Umsatz um einzigartige 18 Prozent steigern. Doch im Laufe der nächsten fünf Jahre brachen die gesamtdeutschen Textilumsätze um 21 Prozent von über 20 Milliarden Euro auf 16,4 Milliarden Euro ein; in der Bekleidungsindustrie reduzierten sich die Umsätze im selben Zeitraum um 3,5 Milliarden Euro auf nur noch 12 Milliarden Euro. Die Beschäftigten traf es noch schlimmer; bundesweit gingen über 60 Prozent der Arbeitsplätze verloren. Während 1990 noch $673.000^{39}$ Beschäftigte in den Textil- und Bekleidungsunternehmen arbeiteten, waren es 1995 nur noch 257.000. Dieser rapide Niedergang eines Industriezweigs war in der deutschen Wirtschaftsgeschichte unvergleichlich.

38 Netzwerk Industrie.Kultur.Ost (o. J.): Die Textilindustrie. Aufstieg \& Fall einer Wirtschaftsmacht.

39 Summe errechnet aus zwei Quellen: Friedrich-Ebert-Stiftung (1992): Textilstandort Ostdeutschland, S. 3 (Quelle für die DDR-Zahlen); Statistisches Bundesamt (Destatis) (2008): Fachserie 4, Reihe 4.1.1 (Quelle für die westdeutschen Zahlen). 
Es dauerte weitere zehn Jahre, bis sich die Situation in beiden Branchen auf niedrigem Niveau stabilisierte. 2010 erwirtschafteten die verbliebenen rund 100.000 Beschäftigten in der Textil- und der Bekleidungsindustrie einen Jahresumsatz von 18 Milliarden Euro. Auch in den Folgejahren blieben die Beschäftigtenzahlen stabil und die Umsatzzahlen schwankten um die 19 Milliarden Euro. Umsatztreiber der Textilindustrie war die Herstellung qualitativ hochwertiger (Chemie)Fasern, also technischer Textilien. Der Bekleidungsindustrie schlug der Wind weiterhin hart ins Gesicht. Sie bekam zunehmend Konkurrenz von vertikal aufgestellten Unternehmen wie H \& M oder Zara, die ihre Prozesse von der Entwicklung bis zum Einzelhandel zunehmend selbst steuerten.

Trotz des enormen Bedeutungsverlustes im Laufe der Jahrzehnte gehört die mittelständisch geprägte Textil- und Bekleidungsindustrie nach wie vor zu den zehn größten Industriezweigen in Deutschland und ist - beide Branchen zusammengenommen - nach dem Ernährungsgewerbe die zweitgrößte Konsumgüterbranche. Sie ist stark in die Fertigung von Nischenprodukten und den Vertrieb von Bekleidung eingebunden und erschließt mit hochwertigen Funktions- und technischen Textilien auch global neue Märkte.

\subsubsection{Die neue internationale Arbeitsteilung - reguliert durch Welttextilabkommen}

Lange bevor »Globalisierung« ein fester Begriff unserer Umgangssprache wurde, gab es den länderübergreifenden Handel mit Textilien oder textilen Vorprodukten. Seide und Baumwolle ergänzten schon im Mittelalter die heimischen Rohstoffe Wolle und Flachs, später dominierte die Baumwolle in der europäischen Textilherstellung. Bald wurden auch andere Waren auf diesen globalen Handelswegen verkauft. Zum Schutz der eigenen Produzenten wurden die nationalen Märkte durch Beschränkungen wie beispielsweise Zölle geschützt.

Nach Ende des Zweiten Weltkriegs waren die Textil- und die Bekleidungsindustrie Pioniere bei der Ausweitung des erneut beginnenden weltweiten Handels. Die Unternehmen in den klassischen Industrieländern mussten sich bereits in den 1950er Jahren mit der globalen Konkurrenz auseinandersetzen. Deshalb forderten die westdeutschen Hersteller schon früh die Beschränkung von Textilund Bekleidungsimporten zum Schutz der Branchen..$^{40}$ Damit stießen sie jedoch auf den Widerstand derer, die mit grenzenlosem Handel steigenden Wohlstand verbanden. Außerdem sollten mit der Aufhebung der Handelshemmnisse vor allem den asiatischen Ländern Exportmöglichkeiten eröffnet werden, um ihre wirtschaftliche Entwicklung zu fördern und sie zugleich »immun« gegen kom-

$40 \mathrm{Vgl}$. Gertschen (2013): Klassenfeinde-Branchenpartner?, S. $63 \mathrm{ff}$. 
munistische Einflüsse zu machen. Bereits 1957 fielen mit der Gründung der Europäischen Wirtschaftsgemeinschaft (EWG) ${ }^{41}$ die ersten Zollschranken in Europa, 1960 folgte die Europäische Freihandelszone EFTA. ${ }^{42}$

Im Gegensatz zur Ausrichtung Europas auf wirtschaftliche Zusammenarbeit sprachen sich die USA dafür aus, den Textilmarkt durch Importquoten zu schützen. 1955 schlossen sie mit Japan das erste internationale Abkommen über den Handel mit Textilien und Bekleidung. Es war als »freiwilliges« Selbstbeschränkungsabkommen konzipiert, hatte aber eine nur eingeschränkte Wirksamkeit. Andere fernöstliche Länder füllten umgehend die auf dem Weltmarkt entstandene Lücke und erhöhten damit zugleich den Angebotsdruck auf die westlichen Hersteller. Die USA reagierten 1961 mit dem sogenannten »Short-Term-Agreement on Cotton Textiles «(STA) zum weltweiten Textil- und Bekleidungshandel. Im Folgejahr wurde das Baumwolltextilabkommen in ein "Long-Term-Agreement « (LTA) umgewandelt und in den Jahren 1967 und 1970 um jeweils drei Jahre verlängert.

Trotzdem blieb die beabsichtigte Schutzwirkung löchrig. Viele Hersteller mischten den Baumwollprodukten andere Fasern bei, so dass es sich nicht mehr um reine Baumwollprodukte handelte, denn mit Mischprodukten konnten die Beschränkungen des Baumwollabkommens umgangen werden. Wieder waren es die USA, die als Erste zum Schutz ihrer Industrie reagierten. Anfang der 1970er Jahre schloss die US-Regierung bilaterale Textilhandel-Beschränkungs-Abkommen mit Japan, Südkorea, Taiwan und Malaysia ab. In der Folge flossen die textilen Warenströme verstärkt in europäische Länder. Mit dieser Taktik gelang es den Amerikanern, das weltweite Baumwollabkommen auf Wolle und Kunstfasern auszuweiten.

Die deutsche Bundesregierung präferierte weiterhin den Freihandel - trotz des von Anfang an heftigen Widerstands der Gewerkschaft Textil-Bekleidung (GTB), die die Arbeitsplätze im Lande sichern und erhalten wollte. Die Befürworter:innen eines uneingeschränkten Handels bezogen sich auf die Theorie von David Ricardo ${ }^{43}$, nach der die Produktion dort am sinnvollsten ist, wo der größte komparative Kostenvorteil möglich ist. Erleichtert wurde die »neue internationale Arbeitsteilung« durch die modernen Transportmöglichkeiten mittels Einführung der Containerwirtschaft. Dadurch entwickelten sich Anfang der 1970er Jahre vor allem Hongkong, Macau, Südkorea und Taiwan zu großen Textilexportnationen.

41 Gründung am 25. März 1957 durch Belgien, Frankreich, Italien, Luxemburg, die Niederlande und die Bundesrepublik Deutschland.

42 Die Europäische Freihandelsassoziation (EFTA) wurde am 4. Januar 1960 durch Dänemark, Norwegen, Österreich, Portugal, Schweden, die Schweiz und das Vereinigte Königreich gegründet. Das Übereinkommen der internationalen Organisation trat am 3. Mai 1960 in Kraft.

43 Vgl. Ricardo (1817): The Principles of Political Economy and Taxation. 


\section{Frau Müller und das Hemd aus Ceylon}

»Der Kauf eines Hemdes aus Ceylon (Sri Lanka) sichert einen Arbeitsplatz in der westdeutschen Textilmaschinenindustrie

Wenn Frau Müller ihrem Mann ein Hemd kauft, dann knüpft sie an dem roten Faden, der sich durch die Weltwirtschaft zieht. Acht von zehn Hemden kommen aus einem Land der Dritten Welt. [...] Teilen heißt: man kann nicht alles haben. Unseren Landsleuten in der Textilindustrie können diese Hemden aus der Dritten Welt Arbeitsplätze wegnehmen. Das ist die eine Seite. Aber an der einfachen Logik, dass andere Länder unsere Maschinen nur bezahlen können, wenn wir ihre Hemden kaufen, an dieser Logik eines freien Welthandels führt kein Weg vorbei.« ${ }^{44}$

So lautete eine Anzeige des Bundeswirtschaftsministers, die im Juli 1978 in zahlreichen deutschen Tageszeitungen erschien. Die bundesdeutsche Regierung unterstrich damit ihren Freihandelsansatz. Eindeutig bevorzugte sie die Investitionsgüterindustrie gegenüber der Herstellung von Konsumgütern. Das war für die damals 570.000 Beschäftigten in der Textil- und Bekleidungsindustrie keine ermutigende Nachricht. Hunderte Betriebsräte schickten Protestschreiben nach Bonn. Darin wurde von Aufforderung zur Arbeitsplatzvernichtung gesprochen, der gleiche Stellenwert für Frauenarbeitsplätze eingefordert wie für den der Männer und es wurde auf die bereits erbrachten Opfer an Beschäftigung verwiesen. Die GTB wies die Bundesregierung darauf hin, dass sich die Näherin in Sri Lanka niemals das von ihr produzierte Hemd kaufen könne, dass in modernsten Fabriken in Asien Hungerlöhne gezahlt würden und die Handels- und Industrieunternehmen der Industrieländer die großen Profite machen. In fast keinem der neuen Textilexportländer würden die Mindeststandards der Internationalen Arbeitsorganisation eingehalten. Außerdem wurde infrage gestellt, ob der Aufbau von Monostrukturen, der neue Abhängigkeiten schaffe, ein wirksamer Beitrag der Entwicklungspolitik sei. ${ }^{45}$

Den Schutz der heimischen Textilproduktion hatten auch Frankreich, Italien sowie die Labour-Regierung Großbritanniens im Sinn, als sie sich vehement für ein erweitertes Handelsbeschränkungsabkommen aussprachen. ${ }^{46}$ Die Gewerkschaf-

44 Zit. nach: Keller (1978): Frau Müller und das Hemd aus Ceylon, S. 3.

45 Keller (1978): Frau Müller und das Hemd aus Ceylon, S. 3 f.

46 Silvia (1990): Jobs, trade and unions, S. 467. 
ten der Industrieländer sahen in den Niedriglöhnen der Entwicklungsländer keinen »natürlichen Kostenvorteil« und kritisierten schon früh die Ausbeutung der dort Beschäftigten. Übrigens waren es auch dort überwiegend Frauen, die an den vielfach urzeitlichen Nähmaschinen saßen.

In den 1970er und 1980er Jahren übte die GTB starken Druck auf die Bundesregierung aus, um den freien Handel mit Ländern mit extrem unterschiedlichen sozialen Verhältnissen zu regulieren. Die Verlagerung der Produktion in Billiglohnländer erhöhe mitnichten den Wohlstand der dortigen Bevölkerung, so dass sie sich die dort hergestellten Waren auch leisten könnten, schließlich ziele diese Form der Industrialisierung ausschließlich auf den Absatz von Exportware. Deshalb werde sich die Spirale der Ausbeutung immer weiterdrehen. Wie recht die GTB mit dieser Einschätzung hatte, zeigt der Blick in die Realität: Der Verlagerung der textilen Produktion nach Süd- und Osteuropa folgte die weitere Verlagerung in Länder mit noch niedrigeren Lohn- und Sozialkosten, nämlich in die Maghreb-Staaten Nordafrikas und vor allem in asiatische Länder. Dass heutzutage ein T-Shirt mitunter weniger kostet als ein Laib Brot, spricht für sich.

Die Hartnäckigkeit der GTB hatte Erfolg und führte zu einem Kurswechsel in Bonn. Die Regierung gab ihren Widerstand auf europäischer Ebene auf und ermöglichte so den Abschluss eines neuen Abkommens. Ende 1973 stimmte sie dem um Chemiefasern erweiterten Multifaserabkommen (MFA) zur Regulierung des internationalen Textilhandels zwischen den Industrie- und Entwicklungsländern zu. Auf dieser Grundlage, die auch mit dem Begriff Welttextilabkommen (WTA) bezeichnet wurde, konnten die Mitgliedsländer von bislang gültigen Handelsregeln abweichen. Die im WTA vereinbarten Quoten und die Beeinträchtigung des Handels mit anderen Ländern durch individuelle Mengenbeschränkungen standen im Widerspruch zu den bestehenden GATT-Prinzipien. ${ }^{47}$ Diese umstrittene Besonderheit war ein einzigartiges Vorgehen, das die Befürworter:innen des unbegrenzten Freihandels zu kritisieren nicht müde wurden.

Beim WTA handelte es sich um ein Rahmenabkommen, das die Leitplanken für bilaterale Selbstbeschränkungsabkommen festlegt. Das Druckmittel der klassischen Industrieländer bestand in Artikel 3 des Abkommens, der im Falle außergewöhnlicher Umstände (»highly unusual and critical circumstances«) einseitige handelsbeschränkende Maßnahmen vorsah. ${ }^{48}$

Der erste auf vier Jahre abgeschlossene Vertrag hatte jedoch einen dramatischen Geburtsfehler: Man ging für die Vertragslaufzeit von viel zu hohen ge-

47 Das Allgemeine Zoll- und Handelsabkommen GATT (General Agreement on Tariffs and Trade) trat 1948 in Kraft und hatte die Förderung der weltwirtschaftlichen Entwicklung und des Wohlstands durch den Abbau von Handelshemmnissen sowie die Schlichtung von Handelskonflikten zum Ziel.

48 Fröbel/Heinrichs/Kreye (1977): Die neue internationale Arbeitsteilung, S. 180. 
samtwirtschaftlichen Wachstumsraten als Grundlage für die Begrenzung der Importquoten aus, so dass das Handelsbeschränkungsabkommen eine jährliche Einfuhrerhöhung um 6 Prozent zuließ. Während der vierjährigen Laufzeit stiegen die Ausgaben der privaten Haushalte für Bekleidung und Schuhe jedoch nur um durchschnittlich 1,2 Prozent pro Jahr. ${ }^{49}$ Schnell verdrängten die preiswerteren Importwaren die im Inland produzierte Ware und der Wert des Importüberschusses stieg von 1973 bis 1976 um 2 Milliarden DM auf 5 Milliarden DM.

Das ab 1978 geltende Folgeabkommen schränkte bei grundsätzlich gleichen Konditionen die Einfuhr einiger hochsensibler Produkte stärker ein, blieb aber weit davon entfernt, die europäische Produktion wirkungsvoll zu schützen. Bereits eineinhalb Jahre vor Auslauf des zweiten WTA signalisierte die EG-Kommission, ${ }^{50}$ maßgeblich beeinflusst durch den deutschen Wirtschaftsminister Otto Graf Lambsdorff, dass sie kein Interesse an einer weiteren Verlängerung habe. Mit vielfältigen Aktionen machten die GTB und ihre europäischen Schwestergewerkschaften deutlich, dass sie diese Entwicklung nicht einfach hinnehmen würden. Es wurden europaweite Arbeitsniederlegungen organisiert, $\mathrm{zu}$ regionalen und landesweiten Demonstrationen aufgerufen. Dies war schließlich der wesentliche Auslöser, dass die stark am Freihandel orientierte Bundesregierung für die Unterstützung der WTA gewonnen werden konnte.

Zum Jahresende 1981 gelang es den gemeinsam agierenden europäischen Textil-Bekleidungs-Gewerkschaften zusammen mit den Textilländern Großbritannien, Frankreich und Italien in letzter Minute, ein neues in mehreren Positionen deutlich stärker einschränkendes Abkommen mit einer Laufzeit von fünf Jahren $\mathrm{zu}$ erreichen. ${ }^{51}$ In der Folgezeit wurde das WTA noch zweimal verlängert.

Im Arbeitgeberlager gab es zu den Handelsbeschränkungen keine einheitliche Auffassung. Etliche Textilunternehmen hatten längst eigene Produktionsstätten in Ländern mit niedrigen Sozial- und Arbeitsstandards aufgebaut und fühlten sich durch die Quoten in ihrer unternehmerischen Freiheit eingeschränkt. Das Schutzbedürfnis anderer europäischer Hersteller empfanden sie als kurzsichtig, zumal sie im Welthandel die größten Gewinnchancen sahen. Dies machte auch Wilhelm Hardt, Präsident von Gesamttextil, deutlich: »Die Wirtschaftspolitik handelt volkswirtschaftlich richtig, wenn sie den natürlichen Strukturwandel [...] nicht zu verhindern sucht.« Er schränkte ein: »Der Staat hat allerdings die Aufga-

49 Quelle: Statistisches Bundesamt (Destatis) (2021): Volkswirtschaftliche Gesamtrechnungen. Bruttoinlandsprodukt, Bruttonationaleinkommen, Volkseinkommen. Lange Reihen ab 1925; eigene Berechnungen.

50 Bis 1967 Europäische Wirtschaftsgemeinschaft (EWG), 1968-1992 Europäische Gemeinschaft (EC), seit 1993 Europäische Union (EU).

51 Silvia (1990): Jobs, trade and unions, S. 507. 
be, die Anpassungsprozesse erträglich zu halten. $\aleph^{52}$ Vehementer Gegner der WTA war die Außenhandelsvereinigung des Deutschen Einzelhandels, die betonte, dass nur ein freizügiger Konsumgüterimport die Konsument:innen mit dem Besten versorge und zugleich die eigene Industrie zu neuen und höheren Leistungen anrege. ${ }^{53}$

Obwohl es mit den Abkommen gelang, die Wirksamkeit für bestimmte Produktgruppen zu erhöhen und damit den Schutz der europäischen Textil- und Bekleidungsindustrie zumindest partiell zu verbessern, vervierfachten sich die entsprechenden Importe von 1973 bis 1992 auf 54 Milliarden DM. Auch wenn die deutschen Unternehmen ihre Exporte erheblich steigerten, wuchs der Importüberschuss in diesem Bereich im gleichen Zeitraum von 5 Milliarden DM auf 24 Milliarden DM (siehe Tabelle 11 im Anhang).

Aber nicht nur Hersteller in den Industrieländern setzten auf Verlagerung der Textilproduktion. Auch die von den WTA-Beschränkungen betroffenen Produktionsländer umgingen ausgeschöpfte Quoten mit der Verlagerung in weitere Niedriglohnländer. Der Kreativität waren keine Grenzen gesetzt, denn die Kontrolle des tatsächlichen Ursprungslandes ist schwierig - einem Hemd sieht man schließlich nicht an, wo es produziert wurde. Zur Umgehung von Exportbeschränkungen errichteten Textilunternehmen neben tatsächlich produzierenden neuen Fabriken in Ländern, die vom Abkommen nicht erfasst wurden, auch virtuell existierende Textilunternehmen. Die textilen Exporte wurden dann von dort oder nur über diese Länder abgewickelt. ${ }^{54}$

Mit dem Auslaufen des fünften WTA wurde 1994 schließlich nach heftigen Diskussionen das "Agreement on Textiles and Clothing " (ATC) unterzeichnet. Damit begann im Folgejahr eine zehnjährige Übergangszeit, an deren Ende der weltweite Handel mit textilen Produkten seine bisherige Sonderstellung im GATT verlor. Ab 2005 galten somit auch für den globalen Textil- und Bekleidungshandel die allgemein gültigen Regeln der Welthandelsorganisation WTO.

Rückblickend muss festgestellt werden, dass die internationalen Textilabkommen den Verlagerungsprozess aus der westlichen Textil- und Bekleidungsindustrie nicht verhindert haben. Aber durch den Schutz vor den überwiegend asiatischen Importen trugen die WTA dazu bei, die Verlagerung der Produktion zeitlich zu strecken.

Die WTA erfassten allerdings nur einen Teil der Importe in die Bundesrepublik, denn es gab auch außerhalb Asiens Wettbewerber mit niedrigeren Lohnkosten. Ein Großteil der textilen Importe kam nach wie vor aus europäischer Pro-

52 Hardt, Wilhelm (1975): Die Textilindustrie im strukturellen Wandel.

53 Außenhandelsvereinigung des Deutschen Einzelhandels: Jahresbericht 1974/75.

54 Vgl. textil-wirtschaft, Ausgabe 27/1976, zit. nach Fröbel/Heinrichs/Kreye (1977): Die neue internationale Arbeitsteilung, S. 181. 
duktion. Mit der Erweiterung der EG auf Portugal und Spanien erhöhte sich 1985 auch die innereuropäische Niedriglohnkonkurrenz und durch die immer wieder erfolgte Abwertung der Lira wurden italienische Textilien für den deutschen Markt preiswerter.

Das für 2005 vereinbarte Ende der Importbeschränkungen löste sowohl in der Gewerkschaft als auch unter Arbeitgebern große Unruhe aus. Die Textil- und die Bekleidungsbranche warnten vor den negativen Auswirkungen auf die heimischen Produktionsstandorte. In der Folge wurden 35.000 Arbeitsplätze abgebaut: Nach 130.000 Beschäftigten im Jahr 2005 stabilisierte sich die Zahl der in den Branchen Beschäftigten ab dem Jahr 2010 bei ca. 100.000. Innerhalb von 40 Jahren gingen somit 92 Prozent der Arbeitsplätze in deutschen Textil- und Bekleidungsunternehmen verloren. Neben den Importen spielten dabei allerdings auch andere Faktoren eine Rolle, nämlich der Wandel im Verbraucherverhalten, der Preisverfall bei Bekleidung und Rationalisierungsmaßnahmen in den Unternehmen. Der Hauptgrund für den gravierenden Arbeitsplatzverlust war jedoch die Verlagerung der Produktion.

\subsection{Die Gewerkschaft Textil-Bekleidung}

Bei den Zusammenschlüssen von Arbeiter:innen in frühindustrieller Zeit waren Textilarbeiter:innen von Beginn an maßgeblich beteiligt. In der Textilindustrie bildeten zunächst die Fabrikarbeiter:innen lokale Zusammenschlüsse, in der Bekleidungsfertigung entstanden die ersten Verbindungen aus der handwerklichen Tradition heraus. Erst nach dem Ende der sogenannten Sozialistengesetze konnten sich in Deutschland ab 1890 reichsweite stabile Gewerkschaften entwickeln. Neben den sogenannten freien, d.h. sozialistisch orientierten Organisationen gründeten sich Gewerkschaftsbünde mit christlich-sozialer und liberaler Ausrichtung.

Nach der Revolution 1918 erlebten die Gewerkschaften eine große Blütezeit, die durch das Inflationsjahr 1923 jäh unterbrochen wurde. Anschließend gelang es auch den Verbänden der Textil- und Bekleidungsarbeiter:innen, sich zu stabilisieren und die Arbeitsbedingungen zu verbessern, bis sie Anfang der 1930er Jahre durch die von der Weltwirtschaftskrise ausgelöste Massenarbeitslosigkeit erneut fundamental geschwächt wurden. 1933 lösten die Nationalsozialisten die Gewerkschaften auf.

Unmittelbar nach der Befreiung von der nationalsozialistischen Gewaltherrschaft wurden lokal wieder freie Gewerkschaften gegründet - jetzt als Einheitsgewerkschaft, in denen alle abhängig Beschäftigten unabhängig von parteipolitischen Präferenzen eine Heimat finden sollten. Die ehemals getrennten 
Gewerkschaften für die Textil- und Bekleidungsbeschäftigten wurden als gemeinsame Organisation wiederaufgebaut.

\subsubsection{Die Vorläuferorganisationen der GTB}

Nach Beginn der Industrialisierung setzte sich die Erkenntnis nur langsam durch, dass sich durch den Zusammenschluss von Arbeitnehmer:innen ein Gegengewicht zur wirtschaftlichen Macht der Fabrikeigentümer schaffen ließ. Insbesondere aus den Arbeiterbildungsvereinen kamen Anregungen zur Gründung von Gewerkschaften. So gründeten die Wolle- und Färbearbeiter:innen in Esslingen im August 1869 mit anderen lokalen Organisationen die »Internationale Gewerksgenossenschaft der Manufaktur-, Fabrik- und Handarbeiter«, in der sich überwiegend Textilarbeiter:innen zusammenschlossen. ${ }^{55}$ Parallel wurden $z u$ dieser Zeit nach den Ideen von Max Hirsch und Franz Duncker die sogenannten HirschDunker'schen Gewerkvereine gegründet, die auf einen Interessenausgleich und die Kooperation von Arbeit und Kapital angelegt waren und der linksliberalen Deutschen Fortschrittspartei nahestanden. In der Textilindustrie stieß dieser Zweig der Gewerkschaftsbewegung jedoch nur auf geringe Resonanz.

Das napoleonische Zivilrecht, das in weiten Teilen Deutschlands auch nach dem Zusammenbruch der napoleonischen Herrschaft weitergalt, enthielt ein Koalitionsverbot. Das Koalitionsrecht, also das Recht auf gemeinsame Interessenvertretung, wurde erst 1869 vom Norddeutschen Bund und 1872 vom Deutschen Reich gewährt. ${ }^{56}$ Doch die gewerkschaftliche Entwicklung stockte während des Deutsch-Französischen Krieges 1870/1871 und kam auch danach kaum voran. Ab 1878 verhinderte das Sozialistengesetz die weitere Festigung der Arbeiterorganisationen und zentrale Verbände konnten sich erst ab 1890, dem Ende der »Verbotszeit«, erneut konstituieren.

Im März 1891 trafen sich 73 Textilarbeiter und 5 Textilarbeiterinnen in Pößneck zum Gründungskongress des »Deutschen Textilarbeiter-Verbandes« (DTAV) - ein Kongress des Hungers und des Elends, wie der Delegierte Clemens Vieweg aus Döbeln ihn nannte. ${ }^{57}$ Einigkeit herrschte bei den Forderungen: Im Vordergrund standen die Einführung des Achtstundentags, das Verbot der Kinderarbeit, ein grundsätzliches Verbot der Nachtarbeit, Ausschluss der Frauenarbeit bei allen den weiblichen Organismus besonders schädigenden Verrichtungen und eine mindestens 36 Stunden umfassende Ruhezeit pro Woche. Der DTAV war sozialistisch ausgerichtet und damit Teil der sogenannten freien Gewerkschaftsbewe-

55 Riebl/Kuhn (1979): Die Anfänge der Gewerkschaften in Esslingen bis 1878, S. 48.

56 Kittner (2005): Arbeitskampf, S. 155 ff.

57 Pößnecker Zeitung, Nr. 75 vom 2.4.1891, Beilage. 
gung mit dem Dachverband »Generalkommission der Gewerkschaften Deutschlands«.

Insbesondere Arbeiter:innen in katholisch geprägten Regionen störten sich an der Religionsfeindlichkeit der sozialistisch geprägten Gewerkschaften. So kam es - schwerpunktmäßig am linken Niederrhein und im Münsterland - zum Zusammenschluss von lokalen christlichen Textilarbeiterverbänden. Am 1. April 1901 wurde der »Zentralverband christlicher Textilarbeiter Deutschlands« gegründet. Wie im DTAV konnten auch hier Frauen Mitglied werden - keine Selbstverständlichkeit, da in dieser Zeit für Frauen ein grundsätzliches Verbot der Mitgliedschaft in politischen Vereinigungen galt. ${ }^{58} 1910$ lag der weibliche Mitgliederanteil der »Christlichen«, die 41.000 Mitglieder hatten, bei 30 Prozent. Der DTAV hatte zu diesem Zeitpunkt 114.000 Mitglieder, der Frauenanteil betrug hier 35 Prozent.

Nach der Gründung der christlichen Gewerkschaften gab es nun - zusammen mit den sozialdemokratisch orientierten freien Gewerkschaften und den HirschDuncker'schen Gewerkvereinen - drei größere Richtungs-Gewerkschaftsbünde. Die Gewerkschaften konkurrierten zwar, stimmten sich aber in der Textilindustrie tarifpolitisch $\mathrm{ab}$ und führten die Arbeitskämpfe hier gemeinsam. ${ }^{59}$

Ein Streik von immenser Bedeutung für die gesamte deutsche Arbeiterbewegung begann am 7. August 1903 in der Textilindustrie von Crimmitschau in Sachsen. 22 Wochen lang kämpften Textilarbeiter:innen um den Zehnstundentag. Dieser erbittert geführte Arbeitskampf erfuhr im ganzen Deutschen Reich Solidarität, wurde aber am 19. Januar 1904 trotz aller Unterstützung bedingungslos abgebrochen. Die Arbeitgeber waren zu keinerlei Zugeständnissen bereit, die Kampfkraft der Gewerkschafter:innen erschöpfte sich zusehends. Im Endeffekt stärkte dieser Arbeitskampf den DTAV. Wie andere Gewerkschaften auch festigte er in der Folge seine Organisation durch höhere Finanzkraft und mehr hauptamtliches Personal. Auf der Arbeitgeberseite war der Crimmitschauer Textilstreik Auslöser zur Gründung des »Vereins deutscher Arbeitgeberverbände«, des Vorläufers der »Bundesvereinigung Deutscher Arbeitgeberverbände« (BDA).

Nach Erstem Weltkrieg, Revolution und Republikgründung erkannten die Arbeitgeber im "Stinnes-Legien-Abkommen«, einem Vertrag zwischen den Spitzenorganisationen der Gewerkschaften und 21 Arbeitgeberverbänden, die Gewerkschaften als legitime Vertretung der Arbeitnehmer:innen an. Die Tarifautonomie wurde garantiert, der Achtstundentag eingeführt und eine »Zentralarbeitsgemeinschaft« mit Branchen-Untergruppen gegründet, in der für die Textil- und Bekleidungsindustrie alle drei Richtungsgewerkschaften mitwirkten.

58 Nach dem preußischen Vereinsrecht war Frauen die Betätigung in Organisationen verboten, die politische Themen beraten. Dieses Verbot wurde am 15. Mai 1908 durch das Reichsvereinsgesetz aufgehoben; vgl. Deutscher Bundestag 2018. 
Damit verbanden sie die Hoffnung, eine Sozialpartnerschaft dauerhaft etablieren $\mathrm{zu}$ können, was sich jedoch als trügerisch erweisen sollte. ${ }^{60}$

Die Blütezeit mit Mitgliederhöchstständen erreichten der DTAV und der 1920 aus verschiedenen Vorläuferorganisationen gegründete "Deutsche Bekleidungsarbeiter-Verband « im Jahr 1922. Der DTAV verzeichnete 738.000 Mitglieder, im Bekleidungsarbeiter-Verband waren 159.000 Beschäftigte organisiert; jeweils rund ein Drittel der Mitglieder waren Frauen. Das Inflationsjahr 1923 zerrüttete die Finanzen der Gewerkschaften: Die von den Mitgliedern in den Betrieben gezahlten Beiträge hatten an Wert verloren, bevor sie verwendet werden konnten, gleichzeitig schmolz der Wert der gewerkschaftlichen Rücklagen. Zu dieser Zeit hatten die Arbeitgeber längst mit einem Rollback begonnen und insbesondere den Achtstundentag angegriffen.

DTAV und Bekleidungsarbeiter-Verband erholten sich in den 1920er Jahren nur schwer von diesen Rückschlägen, die 1924 einen nahezu kompletten Neuaufbau ihrer Organisationen erforderlich machten. Die Mitgliederzahl der Textilgewerkschaft war auf unter 300.000 gesunken; diese Marke wurde erst 1928 wieder überschritten. Die Bekleidungsgewerkschaft hatte in diesem Jahr nur noch knapp 80.000 Mitglieder. ${ }^{61}$

Während der Weltwirtschaftskrise sank die Textilproduktion in Deutschland von 1928 bis 1932 um über 25 Prozent, mit entsprechenden Folgen für die Beschäftigung. ${ }^{62}$ Am 2. Mai 1933 lösten die Nationalsozialisten die freien Gewerkschaften auf. Nach der Ernennung Adolf Hitlers zum Reichskanzler am 30. Januar 1933 begannen allein die Textilarbeiter:innen des kleinen schwäbischen Städtchens Mössingen einen »Generalstreik«, der aber nach wenigen Stunden durch die Polizei aufgelöst wurde. ${ }^{63}$ Die Führung des »Allgemeinen Deutschen Gewerkschaftsbundes « $(A D G B)^{64}$, des Dachverbands der freien Gewerkschaften, hatte am 31. Januar 1933 zwar betont: »Es bedarf keiner Hervorhebung, daß die Gewerkschaften zu dieser Regierung in Opposition stehen«, gleichzeitig aber unterstrichen:

»[...] die Haltung der gewerkschaftlichen Führung kann und darf sich aber nicht von gefühlsmäßigen Gesichtspunkten bestimmen lassen. Daß die deutsche Arbeiterschaft, soweit sie den Geist der deutschen Arbeiterbewegung in sich aufgenommen hat und gewerkschaftlich geschult ist, sich gegen diese sozialreaktionäre Regierung am liebsten in unmittelbarer Aktion zur Wehr setzen würde, ist

60 Vgl. Schönhoven (1980): Wegbereiter der sozialen Demokratie?

61 CTB (1991): textil-bekleidung. 100 Jahre GTB. Sonderausgabe zum Jubiläum, S. 80.

62 Lösch, Hans Peter (1969): Die Textilkonjunktur im allgemeinen Wirtschaftsablauf, S. 35.

63 Vgl. Frick (2015): »Heraus zum Massenstreik«.

64 Der ADCB wurde 1919 als Dachverband der freien, sozialdemokratisch orientierten Cewerkschaften in Nachfolge der »Zentralkommission« gegründet. 
menschlich begreifbar, aber sachlich falsch. [...] Organisation - nicht Demonstration: das ist die Parole der Stunde. $\ll^{65}$

\subsubsection{Gründung der Gewerkschaft Textil-Bekleidung}

Bereits zum Ende der Weimarer Republik gab es Bestrebungen, die drei nach parteipolitischen Präferenzen ausgerichteten Gewerkschaftsbünde zu verschmelzen. Damit wollten die Gewerkschaften der Macht der Arbeitgeber und dem Staat mit vereinter Kraft entgegentreten und insbesondere die demokratische Verfassung der Weimarer Republik verteidigen. Allen Verständigungsbemühungen zum Trotz wurde dieses Ziel erst am 28. April 1933 erreicht, als Hitler bereits drei Monate an der Macht war. Vier Tage später stürmten die Nazis die Gewerkschaftshäuser der freien Gewerkschaften, die Vereinbarung konnte somit nicht mehr umgesetzt werden. ${ }^{66}$ Die christlichen Gewerkschaften unterstellten sich am 3. Mai 1933 dem »Aktionskomitee zum Schutze der deutschen Arbeit« und damit faktisch der nationalsozialistischen »Deutschen Arbeitsfront«, wurden aber trotzdem Ende des Jahres 1933 aufgelöst. ${ }^{67}$

Nach der Befreiung vom Nationalsozialismus war es für die überwältigende Mehrheit der vor 1933 aktiven Gewerkschafter:innen keine Frage, nun »die Einheit zu schaffen", wie es der Gewerkschaftsführer Wilhelm Leuschner vor seiner Hinrichtung durch die Nationalsozialisten am 29. September 1944 nochmals gefordert hatte. Die Einheitsgewerkschaft war keinesfalls nur ein Wunsch der Führungsebene. Dokumente aus dem katholisch geprägten Münsterland, in dem die christliche Textilgewerkschaft bis 1933 nahezu eine Monopolstellung hatte, belegen schon unmittelbar nach Kriegsende den Willen, mit den sozialdemokratischen Gewerkschafter:innen eine gemeinsame Arbeitnehmervertretung aufzubauen. ${ }^{68}$ Zumindest nicht hinderlich war dabei die Tatsache, dass alle Beschäftigten während der nationalsozialistischen Herrschaft zusammen mit den Unternehmern in der »Deutschen Arbeitsfront « in einer Einrichtung zwangsorganisiert waren.

War mit dem Begriff »Einheitsgewerkschaft« in erster Linie die Überwindung der politisch unterschiedlich ausgerichteten Gewerkschaften gemeint, wurde außerdem das Prinzip »Ein Betrieb - eine Gewerkschaft« angestrebt. So wurden politisch und konfessionell unabhängige Einheitsgewerkschaften als fachlich ausgerichtete Verbände gegründet. Eine zunächst angestrebte allgemei-

\footnotetext{
65 12. Bundesausschusssitzung des ADGB am 31. Januar 1933, zit. nach: Gewerkschaftszeitung, Nr. 5/1933, S. 67.

66 Vgl. Kluncker (1999): Der Gedanke der Einheitsgewerkschaft.

67 Hüser (1978): Mit Gott für unser Recht, S. 80.

68 Hüser (1978): Mit Gott für unser Recht, S. 113-117.
} 
ne Einheitsgewerkschaft mit untergeordneten Branchenfachverbänden hatten die Alliierten verhindert und den Aufbau überörtlicher Gewerkschaften durch viele Beschränkungen verzögert. ${ }^{69}$ Auch das Organisationsprinzip "Ein Betrieb - eine Gewerkschaft« konnte nicht ganz verwirklicht werden, da die Deutsche Angestellten-Gewerkschaft (DAG) aus den Vorbereitungen zur Gründung des Deutschen Gewerkschaftsbundes (DGB) ausschied und auch der Deutsche Beamtenbund (DBB) einen eigenen Weg ging. ${ }^{70}$ In der sowjetisch besetzen Zone wurden die Gewerkschaften alsbald dem Monopol der SED-Herrschaft unterstellt.

Für die Textil- und Bekleidungsgewerkschaft war bis zur Vereinigung der Zonen- und Ländergewerkschaften 1949 umstritten, ob der Bereich Leder in die Textil-Bekleidungs-Gewerkschaft mit einbezogen werden sollte. In der britischen Zone war 1947 die Gewerkschaft Textil-Bekleidung-Leder gegründet worden, aber in Süddeutschland entwickelte sich eine eigene Ledergewerkschaft, was sich schließlich als bundesweites Modell durchsetzen sollte.

Noch vor Konstituierung der Bundesrepublik Deutschland beschlossen 160 Delegierte aus den drei Westzonen auf einem Kongress vom 7. bis 9. April 1949 die Gründung der Gewerkschaft Textil-Bekleidung (GTB). Die Auflösung der einzelnen Ländergewerkschaften erfolgte jedoch erst zum 31. Dezember 1949, so dass die vereinigte GTB erst im Januar 1950 auch die Verwaltungs- und Finanzhoheit übernahm. Die sieben Zonen- und Ländergewerkschaften, die sich vereinigten, brachten insgesamt 309.922 Mitglieder ein (siehe Abbildung 4). Obwohl 52 Prozent der Mitglieder Frauen waren, lag ihr Anteil beim Vereinigungskongress mit nur 23 weiblichen Delegierten bei 14,4 Prozent. ${ }^{71}$

69 DCB (1949): Die Gewerkschaftsbewegung in der britischen Zone, S. 42 f.

70 Schönhoven (2014): Geschichte der deutschen Gewerkschaften, S. 70.

71 CTB: Protokoll des Vereinigungskongresses für die Westzonen Deutschlands, 7.-9. April 1949 in Bad Salzuflen, S. 47. 
Abbildung 4: Mitgliederstand der Zonen- und Ländergewerkschaften, die sich zur GTB zusammenschlossen

\begin{tabular}{|l|l|}
\hline britische Zone & 173.738 \\
\hline Bayern & 62.388 \\
\hline Württemberg-Baden & 30.457 \\
\hline Hessen & 14.804 \\
\hline Baden & 11.824 \\
\hline Südwürttemberg-Hohenzollern & 11.352 \\
\hline Rheinland-Pfalz & 5.359 \\
\hline
\end{tabular}

Eigene Darstellung nach: GTB: Ceschäftsbericht 1949-1950 des Hauptvorstandes, S. 5.

Entsprechend der Mitgliederzahl kam die Mehrheit der Delegierten aus der britischen Zone, wo die Gewerkschaft Textil-Bekleidung-Leder schon zwei Jahre zuvor gegründet worden war. Bei den Wahlen setzten sich hauptsächlich deren Vertreter:innen durch: Mit dem Ersten Vorsitzenden Werner Bock und den Vorstandsmitgliedern Bernhard Tacke, Paul Trost, Fritz Knepper und Liesel Kipp-Kaule kamen fünf geschäftsführende Vorstandsmitglieder aus der britischen Zone. Zum Zweiten Vorsitzenden wählten die Delegierten Karl Pöhlmann aus WürttembergBaden, der zuvor gegen Bock um den Vorsitz kandidiert hatte. Pöhlmann trat dieses Amt aber nie an, sondern übernahm die Leitung des vereinigten Bezirks Baden-Württemberg. Somit war der Bayer Hugo Karpf das einzige Vorstandsmitglied, das nicht aus der britischen Zone kam. Er und Tacke gehörten zur christlich-demokratischen Arbeitnehmerschaft.

Neben der Wahl der Führungsgremien war der Gründungskongress von der Behandlung organisatorischer Fragen geprägt: Es war ein Statut zu verabschieden, in dem die Kernaufgaben definiert und der demokratische Aufbau geregelt wurde. Daneben beschlossen die Delegierten nur wenige programmatische Ziele.

Das politische Referat hielt Ludwig Rosenberg vom DGB der britischen Zone. Er betonte die Gleichberechtigung von Kapital und Arbeit und forderte eine Demokratisierung der Wirtschaft. Neben der Sozialisierung der Grundstoffindustrien sollte für alle Betriebe ein Mitbestimmungsrecht der Beschäftigten eingeführt werden. Paritätisch besetzte Wirtschaftskammern sollten Lenkungsaufgaben für die Volkswirtschaft wahrnehmen. Rosenberg betonte, die wirkliche Aufgabe der Gewerkschaft sei nicht der Kampf um bessere Löhne und Arbeitsbedingungen. Dies sei nur der Weg zu einem größeren Ziel, nämlich Menschen zu schaffen, die 
ihr Schicksal selbst bestimmen. ${ }^{72}$ Karl Pöhlmann, der designierte Zweite Vorsitzende, unterstrich in seinem kurzen Schlusswort:

»Wir müssen das kapitalistische System ändern, und ein anderes menschliches und wirtschaftliches System an seine Stelle treten lassen. ${ }^{73}$

Das Statut, wie die erste Satzung bezeichnet wurde, sah drei Ebenen vor (siehe Abbildung 5): Verwaltungsstellen vor Ort, koordinierende Bezirksleitungen, die ein oder mehrere Bundesländer umfassten, und den für die GTB verantwortlichen Hauptvorstand mit einer Hauptvorstandsverwaltung. Der Hauptvorstand wurde vom Gewerkschaftstag gewählt und war diesem gegenüber rechenschaftspflichtig. Der demokratische Aufbau der Organisation blieb im Laufe der Jahrzehnte unverändert. Die Mitglieder wählten in örtlichen Vertreterversammlungen den Verwaltungsstellen-Vorstand sowie die Delegierten für die Bezirkskonferenz und den Gewerkschaftstag. Der Gewerkschaftstag wählte den Hauptvorstand sowie die Mitglieder des Beirats. Die örtlichen Tarifkommissionen und die Mitglieder der Bezirklichen Tarifkommissionen wurden in den jeweiligen Regionen gewählt.

Die Verwaltungsstruktur der GTB bestand aus 91 hauptamtlich besetzten und 135 nebenamtlich organisierten örtlichen Verwaltungsstellen, 9 übergeordneten Bezirksleitungen plus Westberlin mit Sonderstatus und dem Hauptvorstand. ${ }^{74}$ Die Auswahl der Standorte für die zu gründenden örtlichen Büros orientierte sich zunächst an den historisch gewachsenen regionalen Schwerpunkten der Textil- und der Bekleidungsindustrie. Der Sitz des Hauptvorstandes blieb vorerst Bielefeld in der britischen Zone. 1951 wurde er nach Düsseldorf verlegt, wo auch der DGB-Bundesvorstand seine Zentrale hatte. Bei Gründung des Deutschen Gewerkschaftsbundes (DGB) für die Bundesrepublik Deutschland im Oktober 1949 betrug der Mitgliederanteil aus der GTB 6,7 Prozent. ${ }^{75}$ Während ihrer gesamten Existenz setzte sich die GTB für einen starken DGB bei klarer Aufgabenverteilung zwischen Einzelgewerkschaften und Gewerkschaftsbund ein.

72 GTB: Protokoll des Vereinigungskongresses für die Westzonen Deutschlands, 7.-9. April 1949 in Bad Salzuflen, S. 38-43.

73 GTB: Protokoll des Vereinigungskongresses für die Westzonen Deutschlands, 7.-9. April 1949 in Bad Salzuflen, S. 90.

74 GTB: Ceschäftsbericht 1949-1950 des Hauptvorstandes, S. 25 f.

75 DCB: Protokoll des Cründungskongresses, 12.-14. Oktober 1949 in München, S. 281. 
Abbildung 5: Aufbau der Gewerkschaft Textil-Bekleidung (GTB)

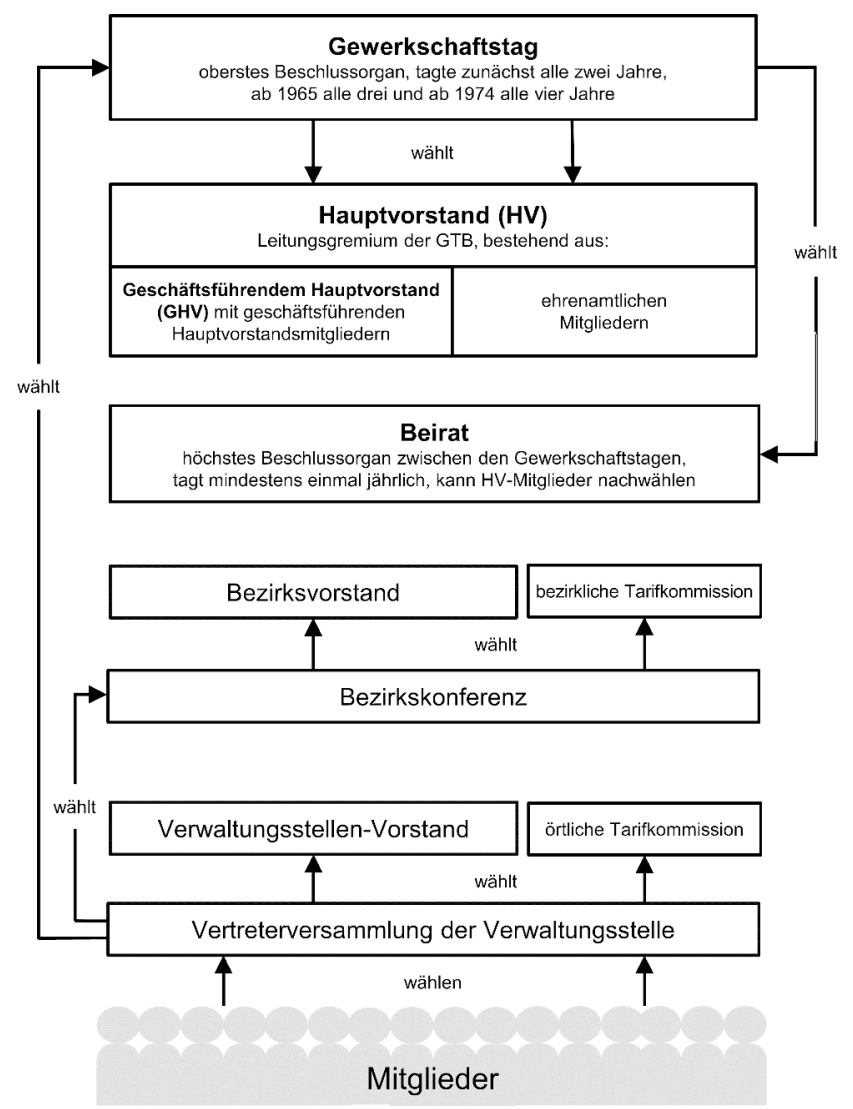

Quelle: Eigene Darstellung.

\subsubsection{Der erste Geschäftsführende Hauptvorstand}

Werner Bock, Jahrgang 1898, gelernter Weber, war früh in der SPD aktiv und wurde 1928 hauptamtlicher Sekretär beim »Deutschen Textilarbeiter-Verband« (DTAV). Später übernahm er die Leitung der Geschäftsstelle in Bielefeld. Bei der Zerschlagung der Gewerkschaften am 2. Mai 1933 wurde er verhaftet und verbrachte mehrere Monate in »Schutzhaft«. Nach seiner Entlassung fand er Arbeit in der Bielefelder Textilindustrie. Später, aus Krieg und Gefangenschaft zurückgekehrt, trieb er zunächst von Bielefeld aus den Aufbau einer Textilgewerkschaft voran und wurde 1947 zum Vorsitzenden der Gewerkschaft Textil-Bekleidung-Leder für die britische Besatzungszone gewählt. 
Als Gewerkschaftsvorsitzender suchte er nicht das Rampenlicht und beschränkte seine Arbeit weitgehend auf die GTB und die internationalen Zusammenschlüsse der Textil- und Bekleidungsgewerkschaften, deren Vereinigung zu einer Internationale er vorantrieb. Im DGB-Bundesvorstand meldete er sich nur selten zu Wort, in politisch strittigen Fragen schlug er sich in der Regel auf die Seite der IG Metall. Mit seiner Skepsis an der Kooperationsbereitschaft der Arbeitgeber galt er auch intern als »Traditionalist«. Innerhalb der GTB war Bock zunächst für Organisation, Personal, Statistik, Jugend und Internationales zuständig.

Bernhard Tacke war von 1928 bis 1933 Sekretär des Zentralverbandes christlicher Textilarbeiter. 1945 gehörte er in Mönchengladbach sowohl zu Gründungsmitgliedern der Textilgewerkschaft wie auch der CDU. Bereits in der britischen Zone war er geschäftsführendes Vorstandsmitglied der Gewerkschaft Textil-Bekleidung-Leder. 1949 wurde er zum Stellvertreter des GTB-Vorsitzenden Werner Bock gewählt und nahm dieses Amt wahr, bis ihn der DGB-Kongress 1956 zum Stellvertretenden Vorsitzenden des Dachverbandes wählte. In der GTB verantwortete er zunächst die Wirtschaftsabteilung und die Tarifpolitik, die 1951 von Karl Buschmann übernommen wurde. Als Christdemokrat war Tacke ein überzeugter Verfechter der Einheitsgewerkschaft. Er stritt für Toleranz gegenüber den christlich-sozial eingestellten Mitgliedern und kritisierte den allgemeinpolitischen Vertretungsanspruch der Gewerkschaften. Dadurch galt er als gemäßigter Gegenspieler von Werner Bock.

Der Hauptkassierer Paul Trost war Sozialdemokrat und vor Beginn der Naziherrschaft im "Zentralverband der Angestellten« aktiv. 1933 wurde er wegen "staatsfeindlicher Tätigkeit« verhaftet. Nach Kriegsende engagierte er sich als Gründungsmitglied der Textilgewerkschaft in Wuppertal und organisierte im Bereich Nordrhein Kassenwesen und innere Verwaltung der neuen Gewerkschaft. Wie Bock und Tacke war er schon in der britischen Zone geschäftsführendes Vorstandsmitglied der Gewerkschaft Textil-Bekleidung-Leder.

Liesel Kipp-Kaule (siehe Kapitel 5.8.1) hatte den Beruf der Schneiderin erlernt. Als weibliches Vorstandsmitglied übernahm sie, wie dies üblich war, die Frauenabteilung der GTB. Zuvor war die Bielefelderin neben ihrer Tätigkeit als geschäftsführendes Vorstandsmitglied der Textilgewerkschaft ehrenamtliches Vorstandsmitglied des DGB für die britische Zone. Für die Gewerkschaftsfrauen war sie Wunschkandidatin für den ersten Geschäftsführenden DGB-Bundesvorstand, wo sie bei der Wahl allerdings unterlag. Im gleichen Jahr wurde sie über die nordrhein-westfälische Landesliste der SPD in den ersten Deutschen Bundestag gewählt. Dem Parlament gehörte sie bis 1965 an. Mit ihrer Branchenerfahrung beeinflusste sie den Ausbau des gesetzlichen Mutterschutzes und den Jugendarbeitsschutz nachhaltig.

Der gelernte Schneider Hugo Karpf war zum Ende der Weimarer Republik Abgeordneter der Bayerischen Volkspartei im Reichstag und gehörte dem Bundestag 
von 1949 bis 1957 als Abgeordneter für die CSU an. Ein Schwerpunkt seiner Arbeit war die Heimarbeitergesetzgebung. In seiner unterfränkischen Heimat, einem Zentrum der deutschen Herrenbekleidungsindustrie, spielte diese Arbeitsform eine große Rolle. Im GTB-Hauptvorstand verantwortete er die Schulungs- und Bildungsarbeit, die Mitgliederwerbung und die Fachgruppe Bekleidung.

Fritz Knepper hatte den Beruf des Seidenwebers erlernt und war ehrenamtlich im DTAV aktiv gewesen. Nach der Machtergreifung der Nationalsozialisten setzte er seine Arbeit illegal fort, was 1938 zu seiner Verhaftung führte. Nach 1945 war er maßgeblich an der lokalen Gründung der Textilgewerkschaft in Haan im Bergischen Land beteiligt, stieg in den Bezirksvorstand und in den Geschäftsführenden Vorstand für die britische Zone auf. Im GTB übernahm der Sozialdemokrat die Verantwortung für Betriebsrätearbeit, Arbeitsrechtsfragen, Sozialpolitik und Angestellte. Zur Zeit der Weimarer Republik hatte Knepper zeitweise der KPD angehört.

\subsection{Die Arbeitgeberverbände}

Im Zuständigkeitsbereich der Gewerkschaft Textil-Bekleidung (GTB) gab und gibt es eine Reihe von Arbeitgeberverbänden und Handwerksinnungen, mit denen die GTB Tarifverträge abgeschlossen hat. 90 Prozent der GTB-Mitglieder wurden jedoch von den Tarifverträgen für die Textil- und Bekleidungsindustrie erfasst, so dass hier eine Beschränkung auf die für diese Branchen zuständigen Verbände erfolgt. Kleinere Branchen mit eigenständigen Tarifverträgen waren insbesondere die Wäschereien, die Bettfedernindustrie, die Schirmindustrie, die Konfektion technischer Textilien, die Polstermöbel- und Matratzenindustrie, das Damenschneiderhandwerk und das Herrenmaßschneiderhandwerk.

\subsubsection{Der "Gesamtverband der Textilindustrie in der Bundesrepublik Deutschland (Gesamttextil)}

Wie im Falle der Gewerkschaften gründeten sich nach der Befreiung auch rasch regionale Unternehmerverbände. Die verschiedenen Sparten der Textilindustrie schlossen sich in der Regel zusätzlich zu Fachverbänden zusammen. Am 25. Mai 1948 gründete sich in der westfälischen Kleinstadt Warendorf die »Arbeitsgemeinschaft der textilindustriellen Verbände des Vereinigten Wirtschaftsgebietes «, ${ }^{76}$ die sich am 10. Februar 1950 in Gesamttextil bzw. »Gesamtverband der Textilindustrie in der Bundesrepublik Deutschland « umbenannte. Erster Präsident wurde der Präsident der Industrie- und Handelskammer Augsburg, Otto A. H.

76 Cesamttextil: Protokoll der Verbandsgründung, 25. Mai 1948 in Warendorf. 
Vogel. Als Sitz des Verbandes bestimmte die Gründungsversammlung Frankfurt am Main.

Im Dachverband waren sowohl die regionalen Arbeitgeberverbände als auch die Fachverbände Mitglied. Gesamttextil war also nicht nur ein Wirtschaftsverband, der die Interessen der Branche gegenüber Politik, Behörden und Öffentlichkeit vertrat, sondern auch ein Arbeitgeberverband, der auf sozial- und tarifpolitischem Gebiet agierte. Der Tarifpolitik kam dabei eine besondere Bedeutung bei, was sich bereits im Juli 1948 mit der Gründung eines "Sozialpolitischen Ausschusses « auch organisatorisch zeigte. ${ }^{77}$

Die Unternehmen waren Mitglieder der regionalen und der Fachverbände von Gesamttextil. Das Machtzentrum bildeten die regionalen Arbeitgeberverbände, bei denen die Unternehmen hauptsächlich ihre Beiträge entrichteten. Die regionalen Arbeitgeberverbände wiederum waren von ihren mittelständischen Mitgliedern geprägt. Beispielsweise waren die typischen Marken der Nachkriegsjahre wie Bleyle, Triumph oder Schiesser seit Generationen in Familienbesitz, selbst wenn sie als Kapitalgesellschaften auftraten. Die patriarchalische Tradition dieser Branche widersprach einer gleichberechtigten Beteiligung von Beschäftigten und darüber hinaus den Mitbestimmungsansprüchen einer Gewerkschaft, deren Repräsentant:innen von außerhalb der »Betriebsfamilie«stammten.

Im November 1950 gab Gesamttextil-Präsident Otto A. H. Vogel seiner drastischen Sicht auf die Gewerkschaften bei einer Kundgebung der »Bundesvereinigung der Deutschen Industrie« (BDI) Ausdruck. Im Kontext der Mitbestimmungsforderungen sprach er vom Gewerkschaftsfunktionär, »der nichts anderes tut, als unproduktiv hin- und herzulaufen, um überall Neid, Missgunst, Unzufriedenheit zu säen und sein Schäfchen ins Trockene zu bringen«. Er sprach den Gewerkschaften das Recht ab, sich den Unternehmern gegenüber »als Sozialpartner aufzuspielen « und verglich die Mitbestimmungsforderungen mit dem Machtanspruch Hitlers. ${ }^{78}$ Wie aus einem Rückblick Vogels vor dem Hauptausschuss von Gesamttextil anlässlich seines Rücktritts vom Präsidentenamt im Jahr 1953 deutlich wird, sahen sich die Unternehmer in der Nachkriegszeit als diejenigen, die nach Kriegsende die Marktwirtschaft durchgesetzt hatten:

»Ich erinnere Sie daran, um was es damals ging: Wohl in erster Linie darum, dass wir einer geradezu diabolisch herrschsüchtigen Bürokratie, die von den Besatzungsmächten allein begünstigte, als demokratisch angesehene kommunistische, sozialistische gewerkschaftliche Arbeiterorganisation nicht ausgeliefert wurden. Es war für uns eine Selbstverständlichkeit, daß der Kampf darum ging: Entweder

77 Gesamttextil: Protokoll zur Gründung des Sozialpolitischen Ausschusses, 23./24. Juli 1948.

78 Vogel (1950): Rede auf der Anti-Mitbestimmungs-Kundgebung »Der deutsche Unternehmer. Leistung und Verpflichtung« des BDI, 8. November 1950 in Köln. 
die Einen oder wir! Das Unglück war, daß der damalige Wirtschaftsdirektor in Minden, Dr. Agartz, ${ }^{79}$ alles getan hat, um die Unternehmer nie mehr in ihre eigentliche Individualsphäre, der Tätigkeit in ihren Betrieben, zurückkehren lassen wollte. Planwirtschaft in eisernen Banden, das war das Ziel und dieses Ziel galt es zu bereinigen. $\ll^{80}$

Dann aber sei Ludwig Erhard gekommen:

»[...] die Hoffnung, die wir uns nie hätten träumen lassen. Nun hatten wir das zu tun, was allein die Möglichkeit bot, um unser Ziel zu erreichen, einerseits Erhard in seiner marktwirtschaftlichen Idee zu unterstützen, ihn aber andererseits gegen seine rot verseuchte Ministerialbürokratie zu schützen. Er ist in diesen Dingen ein außergewöhnlich weicher Mann. ${ }^{81}$

Die erwähnte Unterstützung war nicht nur ideeller Natur: Unmittelbar nach der Gründung von Gesamttextil wurden den bürgerlichen Parteien CDU, CSU und FDP 200.000 Reichsmark als Unterstützung zur Verfügung gestellt. ${ }^{82}$

Wo die Gewerkschaft stark genug war, wurde sie zwangsläufig von den Unternehmern respektiert, doch betrachteten diese die andere Seite nicht als Partner, sondern als Gegner, wenn nicht gar als Feind. So wurden im Juni 1952 in der niederrheinischen Textilindustrie eine Reihe von Betriebsratsmitgliedern entlassen, weil sie sich freigenommen hatten, um an einer Protestkundgebung gegen die Pläne der Bundesregierung für ein Betriebsverfassungsgesetz teilzunehmen. Erst nach einer Intervention der CDU/CSU-Bundestagsfraktion, die durch diese Maßnahme der Textilunternehmer eine Verschärfung der DGB-Proteste befürchtete, wurden die Entlassungen zurückgenommen. ${ }^{83}$

Zur gleichen Zeit beschäftigte sich das Gesamttextil-Präsidium mit der Frage, wie man durch die Unterstützung christlicher Gewerkschaften eine Schwächung der Einheitsgewerkschaften betreiben könne. Diese Linie hielt man im Grundsatz für richtig, allerdings wurde auch Skepsis im Hinblick auf die Erfolgsaussichten geäußert. ${ }^{84}$ Als der Vorsitzende des Handelspolitischen Ausschusses von Gesamttextil, Bernhard van Delden, 1953 mit der GTB-Spitze über wirtschaftspolitische Fragen sprach, wurde er dafür vom Gesamttextil-Präsidium gemaßregelt, nachdem er sich dahingehend geäußert hatte, dass eine Zusammenarbeit mit der GTB

\footnotetext{
79 Siehe Kapitel 3.1.6.

80 Gesamttextil: Hauptausschusssitzung am 20. Februar 1953.

81 Gesamttextil: Hauptausschusssitzung am 20. Februar 1953.

82 Gesamttextil: Beschluss des Präsidiums vom 3. Juni 1948.

83 Gesamttextil: Rundschreiben der Sozialpolitischen Abteilung, 26. Juni 1952.

84 Gesamttextil: Protokoll der Präsidiumssitzung vom 19. Juni 1952.
} 
in Fragen der Außenhandelspolitik erfolgversprechend sein könne. Mündlich wie schriftlich musste er seine »totale Ansichtenänderung«bekunden, wonach er nun überzeugt sei, dass die Einschaltung der Gewerkschaftsspitze zu keinerlei Ergebnissen führen werde. ${ }^{85}$

Gesamttextil und Mitgliedsverbände waren durchaus an der ordnungspolitischen Funktion von Tarifverträgen mit der Gewerkschaft interessiert. Einerseits wurde dadurch die Lohnkonkurrenz zumindest eingeschränkt, andererseits befürchtete man ansonsten eine stärkere Gestaltung durch den Staat. ${ }^{86}$ Zugleich versuchten die großen Player der Branche, ihre Unternehmen aus Tarifkonflikten herauszuhalten. Um Arbeitskämpfe zu vermeiden, wurden Betriebsräten im Vorfeld von als schwierig eingeschätzten Tarifrunden Lohnzugeständnisse gemacht und auch Haustarifverträge abgeschlossen. ${ }^{87}$

Auf die regelmäßigen Streiks - zu Beginn der 1950er Jahre meist betrieblich oder lokal begrenzt, ab 1953 auch ganze Tarifbezirke betreffend - reagierte Gesamttextil mit organisatorischen Veränderungen: Der Sozialpolitische Ausschuss erhielt 1953 größere Autonomie, sein Vorsitzender war nun auch nach außen hin die Vertretung in sozial- und tarifpolitischen Fragen. ${ }^{88}$ Um bestreikte Firmen besser unterstützen zu können, gründete Gesamttextil innerhalb seiner Verbandsstrukturen eine »Gefahrengemeinschaft«. Neben der Zusage organisatorischer Unterstützung durch Arbeitgeberverbände im Falle eines Streiks wurde ein Fonds zur finanziellen Unterstützung bestreikter Firmen gebildet. ${ }^{89}$

1957 wurde der Sozialpolitische Ausschuss zum Arbeitgeberkreis (AGK), ein in der Satzung verankertes Beschlussorgan für die Sozial- und Tarifpolitik. ${ }^{90}$ Vorsitzender wurde der schwäbische Textilunternehmer Hermann Zeiler. Dieser und Carl Neumann, Textilfabrikant aus Wuppertal, der Otto A. H. Vogel 1957 als Präsident von Gesamttextil abgelöst hatte, waren in ihrer Rhetorik zurückhaltender als ihre Vorgänger, aber in ihrem patriarchalischen Selbstverständnis blieb die Gewerkschaft der Störenfried. In der Tarifpolitik für die Textilindustrie waren die Regionalverbände tonangebend; die Koordination über den AGK funktionier-

85 Gesamttextil: Protokoll der Präsidiumssitzung vom 28. April 1953.

86 Dies betonte Hermann Zeiler, der Vorsitzende des Arbeitgeberkreises, anlässlich des »Mannheimer Abkommens « mit der GTB über weitgehende Arbeitszeitverkürzungen; vgl. Gesamttextil: Protokoll der Hauptausschusssitzung vom 1. Februar 1957.

87 Ein Beispiel hierfür ist die Firma Delius in Bielefeld, der Betrieb des Präsidenten des westfälischen Textilverbandes: Bereits Anfang 1953 erhielten die Arbeiter:innen Lohnerhöhungen; als in diesem Tarifbezirk später gestreikt wurde, versuchte die GTB erst gar nicht, in den DeliusBetrieben über einen Streik abstimmen zu lassen; vgl. CTB: Protokoll der Beiratssitzung vom 16./17. Februar 1953, AdsD 5/CTBA0301001.

88 Gesamttextil: Protokoll der Beiratssitzung vom 13. Dezember 1953.

89 Gesamttextil: Protokoll der Beiratssitzung vom 13. Dezember 1953.

90 Gesamttextil: Protokoll der Hauptausschusssitzung vom 11. Dezember 1957. 
te oftmals nur begrenzt. Bis Mitte der 1980er Jahre verweigerten die regionalen Arbeitgeber dem AGK-Geschäftsführer sogar die Teilnahme an Tarifverhandlungen, aber auch danach wichen sie von Absprachen ab, so in den Tarifrunden 1990 und $1994 .{ }^{91}$

1967 begann sich das Verhältnis von Gesamttextil und GTB langsam zu entspannen, vor allem aufgrund der größer werdenden wirtschaftlichen Schwierigkeiten und der politischen Veränderungen, aber auch wegen Versachlichungsangeboten seitens der Gewerkschaft. Seit dem Beginn der ersten Großen Koalition im Jahr 1966 regierten die »natürlichen« Partner von Gesamttextil, CDU/CSU und FDP, nicht mehr allein. Zudem erlebten die Gewerkschaften im Reformklima ab 1968 einen Aufschwung, während das Unternehmerlager gesellschaftspolitisch stärker in die Defensive geriet. Trotz harter tarifpolitischer Auseinandersetzungen verbesserten sich die Kontakte und wurden wirtschaftspolitische Themen besprochen, teilweise kooperierte man auch in der Handelspolitik.

Lange Zeit blieben die Strukturen von Gesamttextil unverändert. Ab 1994 öffneten sich einige Mitgliedsverbände für eine Mitgliedschaft ohne Tarifbindung - eine Zäsur in der Tarifpartnerschaft, die nicht nur in der Textilindustrie zu verzeichnen war. Trotzdem waren die Textilverbände und Gesamttextil grundsätzlich Verteidiger des Flächentarifvertrags. Als es dem AGK-Vorsitzenden Leopold Schoeller, einem Dürener Unternehmer, gemeinsam mit Klaus Schmidt, dem Hauptgeschäftsführer von Gesamttextil, mit dem »Bündnis-Tarifabschluss« von 1996 gelang, Öffnungen in den Tarifverträgen durchzusetzen, akzeptierten die Regionalverbände die neue führende Rolle des Dachverbandes in der Tarifpolitik. Nach der deutschen Wiedervereinigung wurde der Verband der NordOstdeutschen Textilindustrie in den Gesamtverband aufgenommen. Die Tarifpolitik, aber auch die Wirtschaftspolitik für Ostdeutschland wurden vom neuen Mitglied weitgehend allein bearbeitet.

Aufgrund des Schrumpfungsprozesses der Textilindustrie wurden in den 1990er Jahren viele Fachverbände aufgelöst und die Zusammenarbeit mit den Verbänden der Bekleidungsindustrie auf regionaler und zentraler Ebene verstärkt. Mittlerweile gibt es nur noch gemeinsame Verbände: 2002 wurde der »Gesamtverband der deutschen Textil- und Modeindustrie«(Gesamtverband textil+mode) als Nachfolger von Gesamttextil und der Bundesvereinigung Bekleidungsindustrie gegründet. Die als einheitliche Wirtschafts- und Arbeitgeberverbände organisierten Zusammenschlüsse der Textilunternehmer hatten zu allen Zeiten einen hohen Organisationsgrad.

91 So Klaus Schmidt, Hauptgeschäftsführer von Cesamttextil von 1983 bis 2003, in einem Interview am 27. Oktober 2020 mit den Verfasser:innen. 


\subsubsection{Der Bundesverband Bekleidungsindustrie}

Weniger konfrontativ war in den 1950er Jahren das Verhältnis der GTB zum Bundesverband Bekleidungsindustrie (BBI). Verglichen mit der Textilindustrie war der gewerkschaftliche Organisationsgrad in dieser Branche geringer, von den dortigen Arbeitskämpfen des ersten Nachkriegsjahrzehnts blieb man verschont. Die Struktur des BBI war der von Gesamttextil vergleichbar, auch hier waren regionale Arbeitgeberverbände und Fachverbände für einzelne Sparten zusammengeschlossen. Im Unterschied zur Textilindustrie hatten sich die Regionalverbände aber bereiterklärt, bundeseinheitliche Tarifverträge mit der GTB abzuschließen. ${ }^{92}$ Vergleichbar mit dem Arbeitgeberkreis von Gesamttextil wurde innerhalb des BBI die »Bundesvereinigung der Arbeitgeber« mit eigenen Gremien gegründet, der die Zuständigkeit für die Tarif- und Sozialpolitik weitgehend übertragen wurde.

Der BBI sah in Tarifverträgen das zentrale Instrument zur Regelung der Arbeitsbeziehungen in der gesamten Branche, so dass er gemeinsam mit der GTB regelmäßig die Allgemeinverbindlichkeit der Lohn- und Manteltarifverträge beantragte. Das bedeutete, dass deren Tarifregelungen in der Bundesrepublik als Mindestbedingungen für alle Arbeitsverträge der Branche galten. Obwohl in der Bekleidungsindustrie ausschließlich unternehmergeführte Firmen existierten, waren die Arbeitgeberverbände der Bekleidungsindustrie in den ersten Nachkriegsjahrzenten weniger ideologisch geprägt als in der Textilindustrie. Allerdings hatten die Textilindustriellen auch eine viel weiter reichende Firmentradition.

Die Lohnpolitik in der Bekleidungsbranche entwickelte sich zunächst stark im Windschatten der Textilindustrie; die dort erstrittenen Erhöhungsraten wurden regelmäßig nachvollzogen. Wirtschaftlich stand die Bekleidungsindustrie oftmals besser da und verzeichnete in den ersten zwei Jahrzehnten ein höheres und kontinuierlicheres Wachstum als die textile Schwester. Anfang der 1960er Jahre bekam die Tarifpolitik im Windschatten der Textiler einen Bruch. Die GTB drängte die Bekleidungsindustrie in eine Schrittmacherrolle bei Arbeitszeitverkürzung und Urlaubsverlängerung. Dies führte 1962 zum Scheitern der Bundestarifverhandlungen, worauf ein Jahrzehnt lang Tarifverträge nur auf regionaler Ebene abgeschlossen wurden; über neunzehn Jahre hinweg kam es jedoch weder in den Regionen noch auf Bundesebene zu einem neuen Manteltarifvertrag.

In den 1970er Jahren entwickelte sich nach einem Wechsel in der Geschäftsführung des BBI eine intensivere Zusammenarbeit auf der Arbeitsebene. Dies ermöglichte unter anderem eine grundsätzliche Reform des Lohntarifschemas und den Abschluss eines umfassend neu gestalteten Manteltarifvertrags. Diese Ergeb-

92 Die kleinen Regionalverbände für Niedersachsen und die Region Bergisches Land blieben tarifpolitisch selbstständig, ebenso wie das Saarland. 
nisse wurden unabhängig von zunehmenden Differenzen aufgrund der forcierten Auslandsverlagerung der Produktion erreicht. ${ }^{93}$ Der Vorsitzende der Arbeitgebervereinigung, Wilfried Brandes, und der BBI-Hauptgeschäftsführer Rainer Mauer waren auch die Väter des ersten Textil-Bekleidungs-Bündnisses, das die Arbeitgeberverbände 1996 mit der GTB vereinbarten. Mit diesem Vertrag wurde die gemeinsame Tarifpolitik mit den Arbeitgebern der Textilindustrie eingeleitet (siehe Kapitel 7.1.6).

Kurze Zeit später wurden die Bekleidungsverbände selbst Opfer des Abwanderns der Bekleidungsindustrie. Sie schlossen sich den Textilverbänden an, indem der BBI 2002 im Gesamtverband textil+mode aufging. Zuvor waren alle maßgeblichen Unternehmen Mitglied in den Arbeitgeberverbänden des BBI gewesen. Die hohe Tarifbindung hat sich bis in die Gegenwart erhalten.

93 So auch Rainer Mauer, von 1972 bis 1997 Geschäftsführer und später Hauptgeschäftsführer des $\mathrm{BBI}$, in einem Interview am 26. Oktober 2020 mit den Verfasser:innen. 